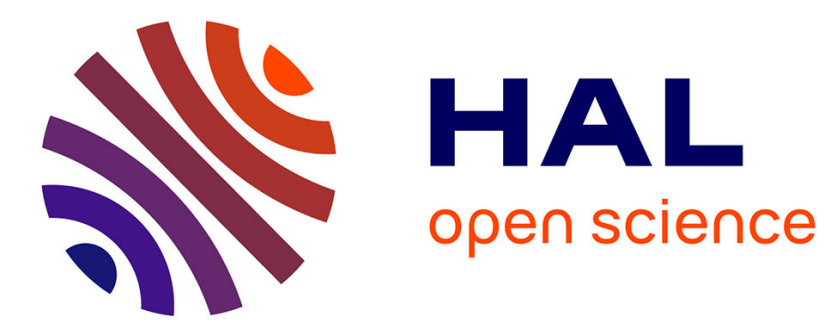

\title{
Divergence-free wavelet projection method for incompressible viscous flow on the square
}

\author{
Souleymane Kadri Harouna, Valérie Perrier
}

\section{To cite this version:}

Souleymane Kadri Harouna, Valérie Perrier. Divergence-free wavelet projection method for incompressible viscous flow on the square. Multiscale Modeling and Simulation: A SIAM Interdisciplinary Journal, 2015, 13 (1), pp.399-422. 10.1137/140969208 . hal-01204929

\section{HAL Id: hal-01204929 \\ https://hal.science/hal-01204929}

Submitted on 24 Sep 2015

HAL is a multi-disciplinary open access archive for the deposit and dissemination of scientific research documents, whether they are published or not. The documents may come from teaching and research institutions in France or abroad, or from public or private research centers.
L'archive ouverte pluridisciplinaire HAL, est destinée au dépôt et à la diffusion de documents scientifiques de niveau recherche, publiés ou non, émanant des établissements d'enseignement et de recherche français ou étrangers, des laboratoires publics ou privés. 


\title{
DIVERGENCE-FREE WAVELET PROJECTION METHOD FOR INCOMPRESSIBLE VISCOUS FLOW ON THE SQUARE
}

\author{
SOULEYMANE KADRI HAROUNA* AND VALÉRIE PERRIER ${ }^{\dagger}$
}

\begin{abstract}
We present a wavelet numerical scheme for the discretization of two-dimensional Navier-Stokes equations with Dirichlet boundary condition on the square. This work is an extension to non periodic boundary conditions of the previous method of Deriaz-Perrier [13]. Here the temporal discretization is borrowed from the projection method. The projection operator is defined through a discrete Helmholtz-Hodge decomposition using divergence-free wavelet bases: this prevents the use of a Poisson solver as in usual methods, whereas improving the accuracy of the boundary condition. The stability and precision order of the new method are stated in the linear case of Stokes equations, confirmed by numerical experiments. Finally the effectiveness, stability and accuracy of the method are validated by simulations conducted on the benchmark problem of lid-driven cavity flow at Reynolds number $R e=1000$ and $R e=10000$.
\end{abstract}

Key words. Divergence-free wavelets, Navier-Stokes simulation, projection method, Dirichlet boundary condition.

1. Introduction. The characterization of turbulent flows is a continuing challenge encountered in several scientific areas. Physically, turbulent flows are characterized by the presence of many phenomena at different scales in interaction and with rapid variations in time and space. The mathematical equations that model turbulent flows are the incompressible Navier-Stokes equations, which are derived from newtonian laws in the context of hydrodynamics [36]:

$$
\left\{\begin{array}{l}
\frac{\partial \boldsymbol{v}}{\partial t}-\nu \Delta \boldsymbol{v}+(\boldsymbol{v} \cdot \nabla) \boldsymbol{v}+\nabla p=0 \\
\nabla \cdot \boldsymbol{v}=0
\end{array}\right.
$$

on $\Omega \subset \mathbb{R}^{d}$, an open domain with smooth or piecewise smooth boundary $\Gamma=\partial \Omega$. Here $\boldsymbol{v} \in \mathbb{R}^{d}$ denotes the velocity vector field, $p \in \mathbb{R}$ is the pressure and $\nu>0$ is the kinematic viscosity. We focus in this paper on the two-dimensional equations $(d=2)$, the extension of our method to dimension three being straightforward.

To take into account the physics of the problem, we suppose that the fluid is confined in $\Omega$, and does not cross the boundary $\Gamma$. In such case, the velocity field $\boldsymbol{v}$ must be tangential to the boundary:

$$
\boldsymbol{v} \cdot \boldsymbol{n}=0 \text { on } \Gamma
$$

where $\boldsymbol{n}$ denotes the outward normal to $\Gamma$. For solid boundary, the viscous flow satisfies a no-slip condition:

$$
\boldsymbol{v}=0 \text { on } \Gamma
$$

whereas a non homogeneous Dirichlet boundary condition:

$$
\boldsymbol{v}=g \text { on } \Gamma
$$

${ }^{*}$ Laboratoire Mathématiques, Image et Applications (MIA), Université de La Rochelle, Avenue Michel Crépeau 17042 La Rochelle, France (souleymane.kadri_harouna@univ-lr.fr).

${ }^{\dagger}$ Laboratoire Jean-Kunztmann, UMR 5224 CNRS, Université de Grenoble-Alpes, 38041 Grenoble Cedex 9, France (valerie.perrier@imag.fr). 
holds for the lid driven cavity test case. More general boundary conditions may be handled, but will not be considered in this article.

The construction of performing numerical schemes is very important for effective models of prediction. The main difficulty in the numerical resolution of Navier-Stokes equations comes from the nature of equations which are nonlinear. Otherwise, physical boundary condition on $\boldsymbol{v}(1.2)$ and (1.3 or 1.4) can be simply imposed considering the velocity-pressure formulation. In such formulation, the projection method has for advantage to decouple the computation of the velocity $\boldsymbol{v}$ and this of the pressure $p$ $[7,35]$.

In this article, we introduce a variant of the projection method based on the Helmholtz-Hodge decomposition [19] of the main equation, explicitly computed in the wavelet domain. This will allow to avoid the principal drawbacks of the classical projection method.

We first introduce the divergence-free function space, with free-slip boundary condition, in order to deal with the incompressibility constraint:

$$
\mathcal{H}_{\text {div }}(\Omega)=\left\{\boldsymbol{u} \in\left(L^{2}(\Omega)\right)^{2}: \nabla \cdot \boldsymbol{u}=0,\left.\boldsymbol{u} \cdot \boldsymbol{n}\right|_{\Gamma}=0\right\} .
$$

By Stokes theorem, the space $\mathcal{H}_{\text {div }}(\Omega)$ is orthogonal to any gradient function in $\left(L^{2}(\Omega)\right)^{2}[19]$. Then, projecting the Navier-Stokes equations $(1.1)$ onto $\mathcal{H}_{\text {div }}(\Omega)$ yields:

$$
\left\{\begin{array}{l}
\frac{\partial \boldsymbol{v}}{\partial t}+\mathbb{P}[-\nu \Delta \boldsymbol{v}+(\boldsymbol{v} \cdot \nabla) \boldsymbol{v}]=0, \\
\nabla \cdot \boldsymbol{v}=0
\end{array}\right.
$$

where $\mathbb{P}$ denotes the orthogonal Leray projector from $\left(L^{2}(\Omega)\right)^{2}$ to $\mathcal{H}_{\text {div }}(\Omega)$. According to the Helmholtz-Hodge decomposition of the term $-\nu \Delta \boldsymbol{v}+(\boldsymbol{v} \cdot \nabla) \boldsymbol{v}$, the pressure should satisfy the following equation:

$$
\nabla p=-\nu \Delta \boldsymbol{v}+(\boldsymbol{v} \cdot \nabla) \boldsymbol{v}-\mathbb{P}[-\nu \Delta \boldsymbol{v}+(\boldsymbol{v} \cdot \nabla) \boldsymbol{v}] .
$$

Now the difficulty relies in the integration in time of (1.6). The conventional projection method consists in a splitting of the operator $\frac{\partial}{\partial t}-\nu \mathbb{P} \Delta$ : a first step computes a -non divergence-free- velocity $\boldsymbol{v}^{*}$ with the operator $\frac{\partial}{\partial t}-\nu \Delta$, followed by a correction step, which consists in the projection of $\boldsymbol{v}^{*}$ onto the divergence-free function space, using a Poisson solver with suitable boundary conditions.

We follow another point of view: in the simplest case of periodic boundary conditions, the first equation of (1.6) becomes:

$$
\frac{\partial \boldsymbol{v}}{\partial t}-\nu \Delta \boldsymbol{v}+\mathbb{P}[(\boldsymbol{v} \cdot \nabla) \boldsymbol{v}]=0
$$

and the pressure $p$ is recovered via:

$$
\nabla p=(\boldsymbol{v} \cdot \nabla) \boldsymbol{v}-\mathbb{P}[(\boldsymbol{v} \cdot \nabla) \boldsymbol{v}] .
$$

Remark that this formulation is very close to the projection method, since the numerical resolution of (1.8) reduces to a heat kernel integration, with as source term the projection of the nonlinear term $\mathbb{P}[(\boldsymbol{v} \cdot \nabla) \boldsymbol{v}]$. This approach is common in spectral 
method [28], where $\mathbb{P}$ is explicit in Fourier domain. It was also used in $[13,14]$, where a divergence-free wavelet based resolution method was introduced. Using a finite difference scheme in time (for example backward Euler), the method is summarized as follows: starting with $\boldsymbol{v}^{n}$, compute $\boldsymbol{v}^{n+1}$ by

$$
(1-\nu \delta t \Delta) \boldsymbol{v}^{n+1}=\boldsymbol{v}^{n}-\delta t \mathbb{P}\left[\left(\boldsymbol{v}^{n} \cdot \nabla\right) \boldsymbol{v}^{n}\right]
$$

where the term $\left(\boldsymbol{v}^{n} \cdot \nabla\right) \boldsymbol{v}^{n}$ is explicitly computed on mesh grid points. Each time step requires the computation of the projection $\mathbb{P}\left[\left(\boldsymbol{v}^{n} \cdot \nabla\right) \boldsymbol{v}^{n}\right]$, which is done through an iterative algorithm using alternatively the div-free and curl-free oblique wavelet projectors [12]. This method gives rise to sparse divergence-free wavelet representation of the velocity, and adaptive discretizations can be derived. Furthermore, one can cite other wavelet-based methods in the velocity-vorticity formulation [16, 30, 32].

Our objective in the next coming sections is to provide an effective numerical method that extends the works of Deriaz-Perrier [13], more flexible for desired boundary conditions. First remark that in the case of physical boundary conditions (1.2) and $(1.3$ or 1.4$)$, the situation becomes more complicated, since the projector $\mathbb{P}$ does not more commute with the Laplacian operator:

$$
\mathbb{P}(\Delta \boldsymbol{v}) \neq \Delta \mathbb{P}(\boldsymbol{v})
$$

Usual approaches consist in computing the divergence of (1.7), which leads to the well known Poisson equation for the pressure $p$ :

$$
\Delta p=\nabla \cdot[(\boldsymbol{v} \cdot \nabla) \boldsymbol{v}]
$$

with Neumann boundary conditions. The resulting equations are then solved by standard methods for heat and Poisson equations, for which a large number of works exist $[19,36]$.

On the other hand, we directly consider equations (1.6-1.7): the spatial approximation will handle divergence-free wavelet bases on square/cubic domains satisfying homogeneous boundary conditions, recently constructed (see $[21,23,33]$ ). In such bases the Helmholtz-Hodge decomposition and the projector $\mathbb{P}$ become explicit and can be computed [22]. We then deduce another formulation of the projection method for Navier-Stokes equations: contrarily to usual approaches [1, 7, 24, 35], the method we develop in next coming sections will not use a Poisson solver.

The paper is organized as follows. Section 2 recalls the setting of divergence-free wavelet bases on the square satisfying homogeneous boundary conditions, and the computation of Leray-Hopf projectors. Section 3 presents the wavelet-based projection method for the unsteady Stokes problem, with a convergence result, validated by numerical experiments. This method is extended for Navier-Stokes equations in Section 4, and compared to benchmark results on the well known lid driven cavity problem.

2. Divergence-free wavelets and Leray-Hopf projector. This section introduces the construction principles and main properties of divergence-free wavelet bases on the square $[0,1]^{2}$, and associated Leray-Hopf projector. Practical details of the construction recalled below can be found in [23]. 
2.1. Divergence-free wavelets. Beginning with the seminal works of LemariéRieusset and collaborators [20, 25], several constructions of divergence-free (and curlfree) wavelets on the square have been provided [23, 33, 34, 37]. All are based on one-dimensional biorthogonal multiresolution analyses linked by differentiation and integration. The construction [23] follows the two following main steps:

(i) Construction of two biorthogonal multiresolution analyses of $L^{2}(0,1)$, denoted by $\left(V_{j}^{1}, \tilde{V}_{j}^{1}\right)$ and $\left(V_{j}^{0}, \tilde{V}_{j}^{0}\right)$ satisfying:

$$
\frac{d}{d x} V_{j}^{1}=V_{j}^{0} \quad \text { and } \quad \tilde{V}_{j}^{0}=\left\{\int_{0}^{x} f(t) d t: f \in \tilde{V}_{j}^{1}\right\} \cap H_{0}^{1}(0,1) .
$$

Each space is spanned by scaling function biorthogonal bases:

$$
V_{j}^{1}=\operatorname{span}\left\{\varphi_{j, k}^{1} ; 0 \leq k \leq N_{j}-1\right\} \text { and } \tilde{V}_{j}^{1}=\operatorname{span}\left\{\tilde{\varphi}_{j, k}^{1} ; 0 \leq k \leq N_{j}-1\right\},
$$

and

$$
V_{j}^{0}=\operatorname{span}\left\{\varphi_{j, k}^{0} ; 0 \leq k \leq N_{j}-2\right\} \text { and } \tilde{V}_{j}^{0}=\operatorname{span}\left\{\tilde{\varphi}_{j, k}^{0} ; 0 \leq k \leq N_{j}-2\right\},
$$

with dimension parameter $N_{j}=2^{j}+c$ (for some small $c \in \mathbb{N}$ ). For $\varepsilon=0,1$, the scaling functions $\varphi_{j, k}^{\varepsilon}$ can be written as $\varphi_{j, k}^{\varepsilon}=2^{j / 2} \varphi^{\varepsilon}\left(2^{j} x-k\right)$ inside the interval $[0,1]$, where $\varphi^{\varepsilon}$ is a compactly scaling function on $\mathbb{R}$, but this is no more true near the boundaries 0 and 1 (idem for $\tilde{\varphi}_{j, k}^{\varepsilon}$ ). In practice, the scale index $j$ must be greater than some index $j_{\min }$, to avoid boundary effects [29]. The biorthogonality between bases writes: $\left\langle\varphi_{j, k}^{\varepsilon} / \tilde{\varphi}_{j, k^{\prime}}^{\varepsilon}\right\rangle=\delta_{k, k^{\prime}}$.

Biorthogonal wavelet spaces $\left(W_{j}^{1}, \tilde{W}_{j}^{1}\right)$ are defined as usual in the biorthogonal wavelet formalism [9]:

$$
W_{j}^{1}=V_{j+1}^{1} \cap\left(\tilde{V}_{j}^{1}\right)^{\perp}, \quad \tilde{W}_{j}^{1}=\tilde{V}_{j+1}^{1} \cap\left(V_{j}^{1}\right)^{\perp} .
$$

These spaces are generated by finite dimensional biorthogonal wavelet bases on the interval:

$$
W_{j}^{1}=\operatorname{span}\left\{\psi_{j, k}^{1} ; 0 \leq k \leq 2^{j}-1\right\} \text { and } \tilde{W}_{j}^{1}=\operatorname{span}\left\{\tilde{\psi}_{j, k}^{1} ; 0 \leq k \leq 2^{j}-1\right\} .
$$

Biorthogonal wavelet bases $\left\{\psi_{j, k}^{0}\right\}_{j \geq j_{\text {min }}}$ of $W_{j}^{0}=V_{j+1}^{0} \cap\left(\tilde{V}_{j}^{0}\right)^{\perp}$ and $\left\{\tilde{\psi}_{j, k}^{0}\right\}_{j \geq j_{\text {min }}}$ of $\tilde{W}_{j}^{0}=\tilde{V}_{j+1}^{0} \cap\left(V_{j}^{0}\right)^{\perp}$ are then simply defined by respectively differentiating and integrating the wavelets bases of $\left(W_{j}^{1}, \tilde{W}_{j}^{1}\right)_{j \geq j_{\text {min }}}[20,23]$ :

$$
\psi_{j, k}^{0}=2^{-j}\left(\psi_{j, k}^{1}\right)^{\prime} \quad \text { and } \quad \tilde{\psi}_{j, k}^{0}=-2^{j} \int_{0}^{x} \tilde{\psi}_{j, k}^{1} .
$$

Homogeneous Dirichlet boundary conditions can be easily imposed on $\left(V_{j}^{1}, \tilde{V}_{j}^{1}\right)$ by removing scaling functions that reproduce constant functions at edges 0 and 1 , prior biorthogonalization $[27,29]$. Then, the spaces

$$
V_{j}^{d}=V_{j}^{1} \cap H_{0}^{1}(0,1)=\operatorname{span}\left\{\varphi_{j, k}^{1} ; 1 \leq k \leq N_{j}-2\right\},
$$

and

$$
\tilde{V}_{j}^{d}=\tilde{V}_{j}^{1} \cap H_{0}^{1}(0,1)=\operatorname{span}\left\{\tilde{\varphi}_{j, k}^{1} ; 1 \leq k \leq N_{j}-2\right\},
$$


provide a biorthogonal multiresolution analysis for $H_{0}^{1}(0,1)$, see [27].

For the construction of divergence-free wavelets in $\left(H_{0}^{1}(\Omega)\right)^{2}$, we will also need to impose homogeneous Neumann boundary conditions to both spaces $V_{j}^{1}$ and $\tilde{V}_{j}^{1}$ :

$$
\begin{aligned}
& V_{j}^{d d}=\left\{f \in V_{j}^{1} ; f(0)=f(1)=0 \text { and } f^{\prime}(0)=f^{\prime}(1)=0\right\}, \\
& \tilde{V}_{j}^{d d}=\left\{f \in \tilde{V}_{j}^{1} ; f(0)=f(1)=0 \text { and } f^{\prime}(0)=f^{\prime}(1)=0\right\} .
\end{aligned}
$$

Following [27, 29], such space can be easily constructed by removing two scaling functions at boundaries, that allows to reconstruct the constant and polynomial of degree 1, then:

$$
V_{j}^{d d}=\operatorname{span}\left\{\varphi_{j, k}^{1} ; 2 \leq k \leq N_{j}-3\right\} \text { and } \tilde{V}_{j}^{d d}=\operatorname{span}\left\{\tilde{\varphi}_{j, k}^{1} ; 2 \leq k \leq N_{j}-3\right\}
$$

It can be proven that: $\frac{d}{d x} V_{j}^{d d} \subset V_{j}^{0 d}$, where $V_{j}^{0 d}=H_{0}^{1} \cap V_{j}^{0}$, which is simply deduced from $V_{j}^{0}$ by removing one scaling functions at each boundary, that not satisfy homogeneous boundary condition. The dual space $\tilde{V}_{j}^{0 d}$ must satisfy an homogeneous Neumann boundary condition to get $\frac{d}{d x} \tilde{V}_{j}^{0 d} \subset \tilde{V}_{j}^{d d}$. The wavelet bases associated to the multiresolution analyses $\left(V_{j}^{d}, \tilde{V}_{j}^{d}\right),\left(V_{j}^{0 d}, \tilde{V}_{j}^{0 d}\right)$ and $\left(V_{j}^{d d}, \tilde{V}_{j}^{d d}\right)$ are constructed using classical approaches [27]. We denote these bases by $\left\{\psi_{j, k}^{d}, \tilde{\psi}_{j, k}^{d}\right\},\left\{\psi_{j, k}^{0 d}, \tilde{\psi}_{j, k}^{0 d}\right\}$ and $\left\{\psi_{j, k}^{d d}, \tilde{\psi}_{j, k}^{d d}\right\}$ respectively and for each basis, a fast wavelet transform algorithm exists with a linear complexity [9, 27, 29, 23].

The approximation order of each space is linked to the number of vanishing moments of its biorthogonal wavelets. Since the space $V_{j}^{1}$ contains polynomials up to degrees $r-1$, it corresponds to an approximation order $r$, and $r$ vanishing moments for the biorthogonal wavelets $\tilde{\psi}_{j, k}^{d}$. Due to the differentiation relation (2.1), the space $V_{j}^{0}$ has for approximation order $r-1$, and $r-1$ vanishing moments for the biorthogonal wavelets $\tilde{\psi}_{j, k}^{0 d}$. Similarly, the wavelets $\psi_{j, k}^{d}$ and $\psi_{j, k}^{0 d}$ have $\tilde{r}$ and $\tilde{r}+1$ vanishing moments respectively.

(ii) Divergence-free wavelet construction

Let $\Omega=[0,1]^{2}$. To construct divergence-free wavelet bases, remark first that the divergence-free space $\mathcal{H}_{\text {div }}(\Omega)$ with free-slip boundary condition (1.5) is the curl of $H_{0}^{1}(\Omega)$ stream functions [19]:

$$
\mathcal{H}_{\text {div }}(\Omega)=\left\{\boldsymbol{u}=\operatorname{curl}(\Psi): \Psi \in H_{0}^{1}(\Omega)\right\} .
$$

By (2.3-2.4) the tensor spaces $\left(V_{j}^{d} \otimes V_{j}^{d}\right)_{j \geq j_{\text {min }}}$ provide a biorthogonal MRA of $H_{0}^{1}(\Omega)$, and divergence-free scaling functions on $\Omega=[0,1]^{2}$ are constructed by taking the curl of scaling functions of $V_{j}^{d} \otimes V_{j}^{d}$ :

$$
\Phi_{j, \boldsymbol{k}}^{d i v}:=\operatorname{curl}\left[\varphi_{j, k_{1}}^{d} \otimes \varphi_{j, k_{2}}^{d}\right]=\mid \begin{aligned}
& \varphi_{j, k_{1}}^{d} \otimes\left(\varphi_{j, k_{2}}^{d}\right)^{\prime} \\
& -\left(\varphi_{j, k_{1}}^{d}\right)^{\prime} \otimes \varphi_{j, k_{2}}^{d}
\end{aligned}, \quad 1 \leq k_{1}, k_{2} \leq N_{j}-2 .
$$


Accordingly, anisotropic divergence-free wavelets on $[0,1]^{2}$ are constructed by taking the curl of the three types of scalar anisotropic wavelets associated to $V_{j}^{d} \otimes V_{j}^{d}$ $\left(j \geq j_{\min }\right)$ :

$\Psi_{\boldsymbol{j}, \boldsymbol{k}}^{d i v, 1}:=\operatorname{curl}\left[\varphi_{j_{m i n}, k}^{d} \otimes \psi_{j_{2}, k_{2}}^{d}\right], \quad \Psi_{\boldsymbol{j}, \boldsymbol{k}}^{d i v, 2}:=\operatorname{curl}\left[\psi_{j_{1}, k_{1}}^{d} \otimes \varphi_{j_{m i n}, k}^{d}\right]$,
$\Psi_{\boldsymbol{j}, \boldsymbol{k}}^{d i v, 3}:=\operatorname{curl}\left[\psi_{j_{1}, k_{1}}^{d} \otimes \psi_{j_{2}, k_{2}}^{d}\right], \quad$ for $\boldsymbol{j}=\left(j_{1}, j_{2}\right), \quad j_{1}, j_{2} \geq j_{m i n}, \quad \boldsymbol{k}=\left(k_{1}, k_{2}\right) \in \mathcal{I}_{\boldsymbol{j}}$,

with:

$$
\mathcal{I}_{\boldsymbol{j}}=\left\{0,1, \ldots, 2^{j_{1}}-1\right\} \times\left\{0,1, \ldots, 2^{j_{2}}-1\right\}
$$

Then, one can prove [23] that:

$$
\begin{aligned}
\mathcal{H}_{\text {div }}(\Omega) & =\operatorname{span}\left\{\Phi_{j_{\text {min }}, \boldsymbol{k}}^{\text {div }} ; \boldsymbol{k}=\left(k_{1}, k_{2}\right) \text { with } 1 \leq k_{1}, k_{2} \leq N_{j_{\text {min }}}-2\right\} \\
& \oplus \operatorname{span}\left\{\Psi_{\boldsymbol{j}, \boldsymbol{k}}^{\text {div } \varepsilon} ; \varepsilon=1,2,3, \boldsymbol{j}=\left(j_{1}, j_{2}\right) \text { with } j_{1}, j_{2} \geq j_{\text {min }}, \boldsymbol{k} \in \mathcal{I}_{\boldsymbol{j}}\right\},
\end{aligned}
$$

which means that each vector function $\boldsymbol{u}$ of $\mathcal{H}_{\text {div }}(\Omega)$ has a unique divergence-free wavelet decomposition:

$$
\boldsymbol{u}=\sum_{\boldsymbol{k}} c_{j_{m i n}, \boldsymbol{k}}^{d i v} \Phi_{j_{m i n}, \boldsymbol{k}}^{d i v}+\sum_{\boldsymbol{j}, \boldsymbol{k}} \sum_{\varepsilon=1,2,3} d_{\boldsymbol{j}, \boldsymbol{k}}^{d i v, \varepsilon} \Psi_{\boldsymbol{j}, \boldsymbol{k}}^{d i v, \varepsilon},
$$

with the norm-equivalence: $\|\boldsymbol{u}\|_{L^{2}}^{2} \sim \sum_{\boldsymbol{k}}\left|c_{j_{m i n}, \boldsymbol{k}}^{\text {div }}\right|^{2}+\sum_{\boldsymbol{j}, \boldsymbol{k}} \sum_{\varepsilon=1,2,3}\left|d_{\boldsymbol{j}, \boldsymbol{k}}^{d i v, \varepsilon}\right|^{2}$, and a linear complexity for the computation of the coefficients [23]. For $j \geq j_{\min }$, the approximation of $\boldsymbol{u}$ on the finite dimensional divergence-free space

$$
\mathbf{V}_{j}^{d i v}=\operatorname{span}\left\{\Phi_{j, \boldsymbol{k}}^{d i v}\right\}_{1 \leq k_{1}, k_{2} \leq N_{j}-2}=\left(V_{j}^{d} \otimes V_{j}^{0}\right) \times\left(V_{j}^{0} \otimes V_{j}^{d}\right) \cap \mathcal{H}_{d i v}(\Omega)
$$

writes using the orthogonal projector:

$$
\mathbb{P}_{j}^{d i v}(\boldsymbol{u})=\sum_{\boldsymbol{k}} c_{j_{m i n}, \boldsymbol{k}}^{d i v} \Phi_{j_{m i n}, \boldsymbol{k}}^{d i v}+\sum_{|\boldsymbol{j}|<j} \sum_{\boldsymbol{k}} \sum_{\varepsilon=1,2,3} d_{\boldsymbol{j}, \boldsymbol{k}}^{d i v, \varepsilon} \Psi_{\boldsymbol{j}, \boldsymbol{k}}^{d i v, \varepsilon}
$$

The approximation error is linked to the approximation order of spaces $V_{j}^{1}$. If spaces $V_{j}^{1}$ contain polynomials up the degree $r-1$, then $V_{j}^{0}$ contain polynomials up the degree $r-2$ and for all $\boldsymbol{u} \in\left(H^{s}(\Omega)\right)^{2}$ with $0 \leq s \leq r-1$, the following Jackson type estimation holds:

$$
\left\|\boldsymbol{u}-\mathbb{P}_{j}^{d i v}(\boldsymbol{u})\right\|_{L^{2}} \leq C 2^{-j s}\|\boldsymbol{u}\|_{H^{s}}
$$

For homogeneous boundary conditions, i.e. in $\left(H_{0}^{1}(\Omega)\right)^{2}$, the divergence-free function space is slightly different, and will be denoted by:

$$
\mathcal{H}_{\text {div }, 0}(\Omega)=\left\{\boldsymbol{u} \in\left(H_{0}^{1}(\Omega)\right)^{2}: \nabla \cdot \boldsymbol{u}=0\right\}=\left(H_{0}^{1}(\Omega)\right)^{2} \cap \mathcal{H}_{\text {div }}(\Omega)
$$

The space $\mathcal{H}_{\text {div }, 0}(\Omega)$ is a closed subspace of $\left(H_{0}^{1}(\Omega)\right)^{2}$, then we have the following decomposition:

$$
\left(H_{0}^{1}(\Omega)\right)^{2}=\mathcal{H}_{\text {div }, 0}(\Omega) \oplus \mathcal{H}_{\text {div }, 0}(\Omega)^{\perp},
$$


which is orthogonal for the scalar product of $\left(H_{0}^{1}(\Omega)\right)^{2}:(\boldsymbol{u}, \boldsymbol{v})_{\left(H_{0}^{1}(\Omega)\right)^{2}}=(\nabla \boldsymbol{u}, \nabla \boldsymbol{v})_{\left(L^{2}(\Omega)\right)^{2}}$. It is easy to prove [19] that:

$$
\mathcal{H}_{\text {div }, 0}(\Omega)^{\perp}=\left\{(-\Delta)^{-1} \nabla q: q \in L^{2}(\Omega)\right\},
$$

where $\boldsymbol{v}=(-\Delta)^{-1} \boldsymbol{f}$ denotes the solution of $-\Delta \boldsymbol{v}=\boldsymbol{f}$, with Dirichlet homogeneous boundary conditions.

Since $\mathcal{H}_{d i v, 0}(\Omega) \subset \mathcal{H}_{d i v}(\Omega)$, a multiresolution analysis of $\mathcal{H}_{d i v, 0}(\Omega)$ is then provided by the spaces:

$$
\mathbf{V}_{j}^{d i v, 0}=\mathbf{V}_{j}^{d i v} \cap\left(H_{0}^{1}(\Omega)\right)^{2}=\left(V_{j}^{d} \otimes V_{j}^{0}\right) \times\left(V_{j}^{0} \otimes V_{j}^{d}\right) \cap\left(H_{0}^{1}(\Omega)\right)^{2} \cap \mathcal{H}_{d i v}(\Omega) .
$$

By definition (2.7) of the space $\mathbf{V}_{j}^{d i v}$, we have $\mathbf{V}_{j}^{d i v, 0}=\operatorname{span}\left\{\Phi_{j, \boldsymbol{k}}^{d i v, 0}\right\}_{2 \leq k_{1}, k_{2} \leq N_{j}-3}$, with:

$$
\Phi_{j, \boldsymbol{k}}^{d i v, 0}:=\operatorname{curl}\left[\varphi_{j, k_{1}}^{d d} \otimes \varphi_{j, k_{2}}^{d d}\right]=\operatorname{curl}\left[\varphi_{j, k_{1}}^{1} \otimes \varphi_{j, k_{2}}^{1}\right], \quad 2 \leq k_{1}, k_{2} \leq N_{j}-3 .
$$

corresponding to scaling functions on $[0,1]$ satisfying both Dirichlet and Neumann boundary conditions at 0 and 1 [29]. Similarly, replacing in the previous construction wavelets of $W_{j}^{d}$ by those of $W_{j}^{d d}$ allows to construct wavelets of $\mathcal{H}_{d i v, 0}(\Omega)$.

Let now $\mathbb{P}_{j}^{d i v, 0}$ be the $L^{2}$-orthogonal projector from $\left(H_{0}^{1}(\Omega)\right)^{2}$ onto $\mathbf{V}_{j}^{d i v, 0}$. Again, for all $\boldsymbol{u} \in\left(H^{s}(\Omega)\right)^{2}$ with $1 \leq s \leq r-1$, the following Jackson type estimation holds, for some $C>0$ :

$$
\left\|\boldsymbol{u}-\mathbb{P}_{j}^{d i v, 0}(\boldsymbol{u})\right\|_{H_{0}^{1}} \leq C 2^{-j(s-1)}\|\boldsymbol{u}\|_{H^{s}} .
$$

2.2. Leray-Hopf projector computation. We present below the basics to compute in practice the Leray-Hopf projector $\mathbb{P}$, using divergence-free wavelet bases, first in the free-slip boundary conditions case (see [22] for more details), then in the case of Dirichlet boundary conditions.

For any vector field $\boldsymbol{u} \in\left(L^{2}(\Omega)\right)^{2}$, the Helmholtz-Hodge decomposition theorem states that, there exist unique $\boldsymbol{u}_{\text {div }} \in \mathcal{H}_{\text {div }}(\Omega)$ and $q \in H^{1}(\Omega)$ with $\int_{\Omega} q=0$, such that:

$$
\boldsymbol{u}=\boldsymbol{u}_{d i v}+\nabla q \quad \text { with } \quad \boldsymbol{u}_{d i v}=\mathbb{P}(\boldsymbol{u})
$$

This decomposition is orthogonal, and $\mathbb{P}:\left(L^{2}(\Omega)\right)^{2} \rightarrow \mathcal{H}_{\text {div }}(\Omega)$ denotes the LerayHopf orthogonal projector. The explicit computation of $\boldsymbol{u}_{\text {div }}$ can be obtained using the divergence-free wavelet basis constructed in Section 2 (with the unified notation $\left.\Psi_{\boldsymbol{j}, \boldsymbol{k}}^{d i v}\right)$ :

$$
\mathcal{H}_{\text {div }}(\Omega)=\operatorname{span}\left\{\Psi_{\boldsymbol{j}, \boldsymbol{k}}^{d i v}\right\}, \quad \forall \boldsymbol{j}, \boldsymbol{k}, \quad \nabla \cdot \Psi_{\boldsymbol{j}, \boldsymbol{k}}^{d i v}=0 \text { and } \Psi_{\boldsymbol{j}, \boldsymbol{k}}^{d i v} \cdot \boldsymbol{n}=0 .
$$

Searching $\boldsymbol{u}_{d i v}$ in terms of its divergence-free wavelet series:

$$
\boldsymbol{u}_{d i v}=\sum_{\boldsymbol{j}, \boldsymbol{k}} d_{\boldsymbol{j}, \boldsymbol{k}}^{d i v} \Psi_{\boldsymbol{j}, \boldsymbol{k}}^{d i v}
$$


and by the orthogonality $\Psi_{\boldsymbol{j}, \boldsymbol{k}}^{\text {div }} \perp \nabla q$ in $\left(L^{2}(\Omega)\right)^{2}$, we obtain:

$$
\left\langle\boldsymbol{u}, \Psi_{\boldsymbol{j}, \boldsymbol{k}}^{\text {div }}\right\rangle=\left\langle\boldsymbol{u}_{\text {div }}, \Psi_{\boldsymbol{j}, \boldsymbol{k}}^{\text {div }}\right\rangle .
$$

Accordingly the computation of coefficients $\left(d_{\boldsymbol{j}, \boldsymbol{k}}^{\text {div }}\right)$ is reduced to the resolution of a linear system:

$$
\mathbb{M}_{d i v}\left(d_{\boldsymbol{j}, \boldsymbol{k}}^{d i v}\right)=\left(\left\langle\boldsymbol{u}, \Psi_{\boldsymbol{j}, \boldsymbol{k}}^{d i v}\right\rangle\right)
$$

where $\mathbb{M}_{\text {div }}$ denotes the Gram matrix of the basis $\left\{\Psi_{\boldsymbol{j}, \boldsymbol{k}}^{\text {div }}\right\}$. The computation of the matrix elements and the right hand side $\left(\left\langle\boldsymbol{u}, \Psi_{\boldsymbol{j}, \boldsymbol{k}}^{d i v}\right\rangle\right)$ in (2.14) is described in [22]. Since the $\Psi_{\boldsymbol{j}, \boldsymbol{k}}^{\text {div }}$ are "curl" functions, the matrix $\mathbb{M}_{\text {div }}$ is no more than the stiffness matrix of the 2D Laplacian operator, on the scalar wavelet basis of $\left(V_{j}^{d} \otimes V_{j}^{d}\right)$ in $H_{0}^{1}(\Omega)$. This matrix, in wavelet basis, admits an optimal (diagonal) preconditioning, and we showed in [22] that the linear system (2.14) is solved with a quasi-linear complexity (with respect to the size of the wavelet coefficient matrix) using a preconditioned conjugate gradient method.

For homogeneous Dirichlet boundary condition problems, we need in this case to compute the $L^{2}$-orthogonal Leray-Hopf projector $\mathbb{P}^{d i v, 0}:\left(H_{0}^{1}(\Omega)\right)^{2} \rightarrow \mathcal{H}_{d i v, 0}(\Omega)$. Since $\mathcal{H}_{d i v, 0}(\Omega)$ is also spanned by a divergence-free wavelets basis (satisfying homogeneous Dirichlet boundary conditions), the computation of $\mathbb{P}^{d i v, 0}$ will be obtained using a similar method than the computation of $\mathbb{P}$.

Let $\left\{\Psi_{\boldsymbol{j}, \boldsymbol{k}}^{d i v, 0}\right\}$ be the divergence-free wavelet basis of $\mathcal{H}_{d i v, 0}(\Omega)$. The $\left(L^{2}(\Omega)\right)^{2}$ orthogonal projector $\mathbb{P}^{d i v, 0}$ is then defined by:

$$
\mathbb{P}^{d i v, 0}(\boldsymbol{u})=\sum_{\boldsymbol{j}, \boldsymbol{k}} d_{\boldsymbol{j}, \boldsymbol{k}}^{d i v, 0} \Psi_{\boldsymbol{j}, \boldsymbol{k}}^{d i v, 0}, \quad \boldsymbol{u} \in\left(H_{0}^{1}(\Omega)\right)^{2},
$$

with

$$
\left(d_{\boldsymbol{j}, \boldsymbol{k}}^{d i v, 0}\right)=\mathbb{M}_{d i v, 0}^{-1}\left(\left\langle\boldsymbol{u}, \Psi_{\boldsymbol{j}, \boldsymbol{k}}^{d i v, 0}\right\rangle_{L^{2}}\right),
$$

and $\mathbb{M}_{d i v, 0}$ denotes the Gram matrix of the basis $\left\{\Psi_{\boldsymbol{j}, \boldsymbol{k}}^{d i v, 0}\right\}$, which is symmetric positive definite.

A $L^{2}$-orthogonal decomposition writes now in $\left(H_{0}^{1}(\Omega)\right)^{2}$ as:

$$
\boldsymbol{u}=\boldsymbol{u}_{d i v, 0}+\boldsymbol{u}_{d i v, 0}^{\perp} \quad \text { with } \quad \boldsymbol{u}_{d i v, 0}=\mathbb{P}^{d i v, 0}(\boldsymbol{u})
$$

The orthogonal part $\boldsymbol{u}_{d i v, 0}^{\perp}=u-\mathbb{P}^{d i v, 0}(\boldsymbol{u})$ satisfies, for all $\boldsymbol{v} \in \mathcal{H}_{d i v, 0}(\Omega)$ :

$$
\left\langle\boldsymbol{u}-\mathbb{P}^{d i v, 0}(\boldsymbol{u}), \boldsymbol{v}\right\rangle_{L^{2}}=\langle\boldsymbol{u}, \boldsymbol{v}\rangle_{L^{2}}-\left\langle\mathbb{P}^{d i v, 0}(\boldsymbol{u}), \boldsymbol{v}\right\rangle_{L^{2}}=\langle\boldsymbol{u}, \boldsymbol{v}\rangle_{L^{2}}-\left\langle\boldsymbol{u}, \mathbb{P}^{d i v, 0}(\boldsymbol{v})\right\rangle_{L^{2}}=0,
$$

Using De Rhams theorem [19], there exists $p \in H^{1}(\Omega)$ such that $\boldsymbol{u}-\mathbb{P}^{d i v, 0}(\boldsymbol{u})=\nabla p$. Every function $\boldsymbol{u} \in\left(H_{0}^{1}(\Omega)\right)^{2}$ can be decomposed as:

$$
\boldsymbol{u}=\mathbb{P}^{d i v, 0}(\boldsymbol{u})+\left(\boldsymbol{u}-\mathbb{P}^{d i v, 0}(\boldsymbol{u})\right)=\mathbb{P}^{d i v, 0}(\boldsymbol{u})+\nabla p .
$$

where $\nabla p$ satisfies homogeneous boundary conditions. 
3. Modified projection method based on divergence-free wavelets. The purpose in this section is to introduce a wavelet based numerical scheme for incompressible viscous flows calculations. The method can be seen as a variant of the projection method $[1,7,24,35]$, where we replace the operator splitting by the exact Helmholtz-Hodge decomposition of the intermediate velocity field, computed using divergence-free wavelets. Then, we prevent some numerical difficulties and drawbacks related to the computation of the pressure at each time step with artificial boundary conditions done in the classical approaches [1,24].

3.1. General principles of divergence-free wavelet schemes for the Stokes equations. The use of divergence-free wavelet bases in incompressible flow calculations began with the works of Urban et al [11,37], for the resolution of the stationary Stokes problem:

$$
\left\{\begin{array}{l}
-\nu \Delta \boldsymbol{v}+\nabla p=\boldsymbol{f} \\
\nabla \cdot \boldsymbol{v}=0
\end{array}\right.
$$

in $\Omega=[0,1]^{2}$, with periodic or homogeneous Dirichlet boundary conditions.

The main advantage of using divergence-free wavelet basis in the resolution of Stokes equations is the direct representation of the incompressibility constraint of the flow. Following Urban's works [37, 39] problem (3.1) is solved using a variational approach, and a Galerkin approximation using divergence-free wavelets as trial functions. The velocity field $\boldsymbol{v}$ is searched in terms of its divergence-free wavelet coefficients:

$$
\boldsymbol{v}(x)=\sum_{\boldsymbol{j}, \boldsymbol{k}} d_{\boldsymbol{j}, \boldsymbol{k}} \Psi_{\boldsymbol{j}, \boldsymbol{k}}^{d i v}(x) .
$$

Replacing (3.2) into (3.1), the computation of coefficients $d_{\boldsymbol{j}, \boldsymbol{k}}$ is done by solving the linear system:

$$
\nu \sum_{\boldsymbol{j}, \boldsymbol{k}} d_{\boldsymbol{j}, \boldsymbol{k}}\left\langle\nabla \Psi_{\boldsymbol{j}, \boldsymbol{k}}^{d i v}, \nabla \Psi_{\boldsymbol{j}^{\prime}, \boldsymbol{k}^{\prime}}^{d i v}\right\rangle=\left\langle\boldsymbol{f}, \Psi_{\boldsymbol{j}^{\prime}, \boldsymbol{k}^{\prime}}^{d i v}\right\rangle, \quad \forall \boldsymbol{j}^{\prime}, \boldsymbol{k}^{\prime}
$$

The divergence-free wavelet stiffness matrix $\left[\left\langle\nabla \Psi_{\boldsymbol{j}, \boldsymbol{k}}^{\text {div }}, \nabla \Psi_{\boldsymbol{j}^{\prime}, \boldsymbol{k}^{\prime}}^{\text {div }}\right\rangle\right]$ is symmetric and the associated bilinear form is coercive [37]. The problem is thus reduced to an elliptic problem on some finite-dimensional divergence-free function space $\mathbf{V}_{j}^{\text {div }}$ : therefore standard error estimations hold, derived from the projection error $(2.9,2.11)$. In addition, the formulation (3.3) has the advantage to eliminate directly the pressure $p$ which is computed by a post processing procedure [37].

In comparison with classical approaches based on finite differences, finite elements or (non divergence-free) wavelet methods [5, 19], equation (3.3) presents the advantage of reducing the number of degree of freedom: only-scalar-coefficients $\left\{d_{\boldsymbol{j}, \boldsymbol{k}}\right\}$ are computed instead of one type of coefficients per components of the velocity $\boldsymbol{v}$. Moreover, adaptive strategies can be applied to only compute significant wavelet coefficients, and optimal preconditioning for the stiffness matrix can be provided explicitly [8, 22].

For unsteady problems, recently Stevenson [33] proposed a new theoretical variational formulation of the Stokes equations. The method of Stevenson is an extension 
of Urban's method to the unsteady problem using divergence-free wavelets satisfying a free-slip boundary condition.

We adopt in the present article another point of view, and the method we present in next coming sections will use a Galerkin formulation with standard wavelet bases for the approximation of the velocity, and the Leray-Hopf projector $\mathbb{P}$ computed with divergence-free wavelets. This leads to a new projection method formulation, using divergence-free wavelets satisfying Dirichlet boundary condition. The advantage is that classical wavelet methods can be used to solve the diffusion problem and the incompressibility constraint is incorporated via the projector $\mathbb{P}$. The approach will include one phase devoted to the temporal discretization and a second one to spatial discretization.

3.2. Modified projection method for unsteady Stokes equations. We consider in this section the unsteady Stokes problem, with no-slip boundary conditions:

$$
\left\{\begin{array}{l}
\frac{\partial \boldsymbol{v}}{\partial t}-\nu \Delta \boldsymbol{v}+\nabla p=\boldsymbol{f} \\
\boldsymbol{v}=0 \text { on } \partial \Omega \\
\nabla \cdot \boldsymbol{v}=0
\end{array}\right.
$$

The time discretization of system (3.4) is obtained through a finite difference method. Without loss of generality, given a time step $\delta t$ and considering the approximation $\boldsymbol{v}^{n}(x) \approx \boldsymbol{v}(x, n \delta t)$, the backward Euler scheme leads to:

$$
\frac{\boldsymbol{v}^{n+1}-\boldsymbol{v}^{n}}{\delta t}-\nu \Delta \boldsymbol{v}^{n+1}+\nabla p^{n+1}=\boldsymbol{f}^{n}, \quad \nabla \cdot \boldsymbol{v}^{n+1}=0
$$

However, scheme (3.5) is inefficient since it requires, at each time step, the solution of coupled equations for $\left(\boldsymbol{v}^{n+1}, p^{n+1}\right)$.

Now, let us introduce new variables $\tilde{\boldsymbol{v}}^{n} \in\left(H_{0}^{1}(\Omega)\right)^{2}$ and $\Phi^{n}$ by setting $\tilde{\boldsymbol{v}}^{n+1}=$ $\boldsymbol{v}^{n+1}+\nabla \Phi^{n+1}$. One can prove that $\tilde{\boldsymbol{v}}^{n+1}$ verifies the following system:

$\frac{\tilde{\boldsymbol{v}}^{n+1}-\boldsymbol{v}^{n}}{\delta t}-\nu \Delta \tilde{\boldsymbol{v}}^{n+1}+\nabla\left[p^{n+1}-\frac{1}{\delta t} \Phi^{n+1}+\nu \Delta \Phi^{n+1}\right]=\boldsymbol{f}^{n}, \quad \boldsymbol{v}^{n+1}=\mathbb{P}^{\operatorname{div}, 0}\left(\tilde{\boldsymbol{v}}^{n+1}\right)$.

This equation can be split by defining the pressure $p^{n+1}$ such that:

$$
p^{n+1}-\frac{1}{\delta t} \Phi^{n+1}+\nu \Delta \Phi^{n+1}=0,
$$

and (3.6) reduces to

$$
(1-\nu \delta t \Delta) \tilde{\boldsymbol{v}}^{n+1}=\boldsymbol{v}^{n}+\delta t \boldsymbol{f}^{n}, \quad \boldsymbol{v}^{n+1}=\mathbb{P}^{d i v, 0}\left(\tilde{\boldsymbol{v}}^{n+1}\right) .
$$

Remark that the change of variable of this formulation is similar to a Gauge formulation applied to the discrete equation (3.5). The pressure is replaced by a Gauge variable $\Phi$, linked to $p$ by equation (3.7), which replaces a continuous heat equation appearing in the continuous formulation [40]. However, the difference between our method and the Gauge method lies in the choice of boundary conditions. Instead 
of imposing $\nabla \Phi^{n+1} \cdot \boldsymbol{\tau}=0$ or $\nabla \Phi^{n+1} \cdot \boldsymbol{n}=0$ as in the Gauge method, we choose boundary conditions on the auxiliary field $\tilde{\boldsymbol{v}}^{n+1}$ equal to those of $\boldsymbol{v}^{n}$, which leads to:

$$
\tilde{\boldsymbol{v}}^{n+1}=0 \text { and } \nabla \Phi^{n+1}=0, \text { on } \partial \Omega,
$$

then we get the desired boundary conditions $\boldsymbol{v}^{n}=0$ on $\partial \Omega$ (note that in our method $\Phi$ has no physical meaning).

The resolution of (3.8) requires the resolution of a heat equation followed by a projection step, described in Section 2.2. The spacial discretization is obtained through a variational Galerkin method on suitable tensorial wavelet bases.

3.3. Spatial discretization of Stokes equations. The spatial approximation of the velocity will be performed using the multiresolution analysis of $\left(H_{0}^{1}(\Omega)\right)^{2}$ that contains the divergence-free wavelets. This multiresolution analysis is constituted by the vector spaces $\mathbf{V}_{j}^{d}=\left(V_{j}^{d} \otimes V_{j}^{0 d}\right) \times\left(V_{j}^{0 d} \otimes V_{j}^{d}\right)$.

We now consider a Galerkin formulation of equations (3.8): at a given resolution $j$, the components of the approximate solution $\boldsymbol{v}_{j}^{n}=\left(v_{1, j}^{n}, v_{2, j}^{n}\right)$ are searched under the form of finite dimensional wavelet series in $\mathbf{V}_{j}^{d}$ :

$$
v_{1, j}^{n}=\sum_{|\boldsymbol{j}|<j, \boldsymbol{k}} d_{\boldsymbol{j}, \boldsymbol{k}}^{1, n} \psi_{j_{1}, k_{1}}^{d} \otimes \psi_{j_{2}, k_{2}}^{0 d} \text { and } v_{2, j}^{n}=\sum_{|\boldsymbol{j}|<j, \boldsymbol{k}} d_{\boldsymbol{j}, \boldsymbol{k}}^{2, n} \psi_{j_{1}, k_{1}}^{0 d} \otimes \psi_{j_{2}, k_{2}}^{d},
$$

and similarly for $\tilde{\boldsymbol{v}}_{j}^{n}$ with coefficients $\left[\tilde{d}_{\boldsymbol{j}, \boldsymbol{k}}^{1, n}\right]$ and $\left[\tilde{d}_{\boldsymbol{j}, \boldsymbol{k}}^{2, n}\right]$.

Denoting by $a_{\delta t}$ the bilinear form:

$$
a_{\delta t}(\boldsymbol{v}, \boldsymbol{w})=\int_{\Omega} \boldsymbol{v} \boldsymbol{w}+\nu \delta t \int_{\Omega} \nabla \boldsymbol{v} \cdot \nabla \boldsymbol{w}
$$

and by $\mathbb{P}_{j}^{d i v, 0}$ the restriction of the Leray projector $\mathbb{P}^{d i v, 0}$ to $\mathbf{V}_{j}^{d}$, the Galerkin formulation of equations (3.8) writes at each time-step $n$ : knowing the approximate solution $\boldsymbol{v}_{j}^{n} \in \mathbf{V}_{j}^{d i v, 0}$ and the r.h.s. $f_{j}^{n} \in \mathbf{V}_{j}^{d}$, find $\tilde{\boldsymbol{v}}_{j}^{n+1} \in \mathbf{V}_{j}^{d}, \boldsymbol{v}_{j}^{n+1} \in \mathbf{V}_{j}^{d i v, 0}$ s.t. $\forall \boldsymbol{w}_{j} \in \mathbf{V}_{j}^{d}$,

$$
a_{\delta t}\left(\tilde{\boldsymbol{v}}_{j}^{n+1}, \boldsymbol{w}_{j}\right)=\int_{\Omega} \boldsymbol{v}_{j}^{n} \boldsymbol{w}_{j}+\delta t \int_{\Omega} \boldsymbol{f}_{j}^{n} \boldsymbol{w}_{j}, \quad \boldsymbol{v}_{j}^{n+1}=\mathbb{P}_{j}^{d i v, 0}\left(\tilde{\boldsymbol{v}}_{j}^{n+1}\right) .
$$

Now the tensorial structure of wavelet bases of $\mathbf{V}_{j}^{d}$ allows to factorize at each time step the stiffness matrix of the discrete heat operator $(1-\delta t \nu \Delta)$, like in [6]. Such factorization remains in the context of alternated direction implicit (ADI) methods, and consists, for small $\alpha$, to use the approximation:

$$
(1-\alpha \Delta) \approx\left(1-\alpha \frac{\partial^{2}}{\partial x^{2}}\right)\left(1-\alpha \frac{\partial^{2}}{\partial y^{2}}\right) .
$$

Remark that this ADI factorization (3.12) neglects the fourth order derivatives and this has some implication for the unsteady boundary conditions. In such case, the spatial discretization accuracy has to be improved by increasing the number of vanishing moments of the wavelet family. In this work we will only consider steady Dirichlet boundary condition.

Thus, in (3.11) we only have to invert the Galerkin matrix of the one-dimensional heat operator $\left(1-\nu \delta t \frac{\partial^{2}}{\partial x^{2}}\right)$, which is done once before starting the time integration 
procedure. Finally, the computation of matrices of coefficients $\left[\tilde{d}_{\boldsymbol{j}, \boldsymbol{k}}^{, n+1}\right]$ and $\left[\tilde{d}_{\boldsymbol{j}, \boldsymbol{k}}^{2, n+1}\right]$ from those of $\boldsymbol{v}^{n}$ reduces to solve the following systems:

$$
\mathcal{A}_{\delta t}^{d}\left[\tilde{d}_{\boldsymbol{j}, \boldsymbol{k}}^{1, n+1}\right] \mathcal{A}_{\delta t}^{0 d}=\mathcal{M}^{d}\left[d_{\boldsymbol{j}, \boldsymbol{k}}^{1, n}\right] \mathcal{M}^{0 d}+\delta t \mathcal{M}^{d} \boldsymbol{f}_{j 1}^{n} \mathcal{M}^{0 d}
$$

and

$$
\mathcal{A}_{\delta t}^{0 d}\left[\tilde{d}_{\boldsymbol{j}, \boldsymbol{k}}^{2, n+1}\right] \mathcal{A}_{\delta t}^{d}=\mathcal{M}^{0 d}\left[d_{\boldsymbol{j}, \boldsymbol{k}}^{2, n}\right] \mathcal{M}^{d}+\delta t \mathcal{M}^{0 d} \boldsymbol{f}_{j 2}^{n} \mathcal{M}^{d},
$$

where $\mathcal{A}_{\delta t}^{\varepsilon}$ and $\mathcal{M}^{\varepsilon}$ correspond respectively to the stiffness matrix of operator $(1-$ $\left.\nu \delta t \frac{\partial^{2}}{\partial x^{2}}\right)$ and the Gram matrix of the one-dimensional wavelet basis of $\left\{V_{j}^{\varepsilon}\right\}_{\varepsilon=d, 0 d}$ :

$$
\mathcal{A}_{\delta t}^{d}=\left[\left\langle\psi_{j, k}^{d}, \psi_{j^{\prime}, k^{\prime}}^{d}\right\rangle+\nu \delta t\left\langle\left(\psi_{j, k}^{d}\right)^{\prime},\left(\psi_{j^{\prime}, k^{\prime}}^{d}\right)^{\prime}\right\rangle\right] \text { and } \mathcal{M}^{d}=\left[\left\langle\psi_{j, k}^{d}, \psi_{j^{\prime}, k^{\prime}}^{d}\right\rangle\right],
$$

and

$$
\mathcal{A}_{\delta t}^{0 d}=\left[\left\langle\psi_{j, k}^{0 d}, \psi_{j^{\prime}, k^{\prime}}^{0 d}\right\rangle+\nu \delta t\left\langle\left(\psi_{j, k}^{0 d}\right)^{\prime},\left(\psi_{j^{\prime}, k^{\prime}}^{0 d}\right)^{\prime}\right\rangle\right] \text { and } \mathcal{M}^{0 d}=\left[\left\langle\psi_{j, k}^{0 d}, \psi_{j^{\prime}, k^{\prime}}^{0 d}\right\rangle\right]
$$

Elements of the form $\left\langle\psi_{j, k}^{d}, \psi_{j^{\prime}, k^{\prime}}^{d}\right\rangle$ or $\left\langle\left(\psi_{j, k}^{d}\right)^{\prime},\left(\psi_{j^{\prime}, k^{\prime}}^{d}\right)^{\prime}\right\rangle$ are analytically computed, by solving eigenvalue problems [2, 29].

We summarize below the resolution algorithm. Starting with an initial velocity $\tilde{\boldsymbol{v}}_{0}(\boldsymbol{x})=\boldsymbol{v}_{0}(\boldsymbol{x})=\boldsymbol{v}(0, \boldsymbol{x})$, compute its wavelet coefficients $\left[\tilde{d}_{\boldsymbol{j}, \boldsymbol{k}}^{1,0}\right]$ and $\left[\tilde{d}_{\boldsymbol{j}, \boldsymbol{k}}^{2,0}\right]$ in $\mathbf{V}_{j}^{d}[21]$. For $1 \leq n \leq N$, repeat:

Step 1: Find $\left[\tilde{d}_{\boldsymbol{j}, \boldsymbol{k}}^{1, n+1}\right]$ and $\left[\tilde{d}_{\boldsymbol{j}, \boldsymbol{k}}^{2, n+1}\right]$ solution of $(3.13,3.14)$.

Step 2: Find $\left[\boldsymbol{d}_{\boldsymbol{j}, \boldsymbol{k}}^{d i v, n+1}\right]$ solution of

$$
\mathcal{M}^{d}\left[d_{\boldsymbol{j}, \boldsymbol{k}}^{d i v, n+1}\right] \mathcal{R}^{d}+\mathcal{R}^{d}\left[d_{\boldsymbol{j}, \boldsymbol{k}}^{d i v, n+1}\right] \mathcal{M}^{d}=\mathcal{M}^{d}\left[\tilde{d}_{\boldsymbol{j}, \boldsymbol{k}}^{1, n+1}\right] \mathcal{A}_{d}^{0}-\left(\mathcal{A}_{d}^{0}\right)^{T}\left[\tilde{d}_{\boldsymbol{j}, \boldsymbol{k}}^{2, n+1}\right] \mathcal{M}^{d},
$$

where $\mathcal{R}^{d}=\left[\left\langle\left(\psi_{j, k}^{d}\right)^{\prime},\left(\psi_{j^{\prime}, k^{\prime}}^{d}\right)^{\prime}\right\rangle\right]$ and $\mathcal{A}_{d}^{0}=\left[\left\langle\psi_{j, k}^{0 d},\left(\psi_{j^{\prime}, k^{\prime}}^{d}\right)^{\prime}\right\rangle\right][22]$.

Step 3: Compute $\left[d_{\boldsymbol{j}, \boldsymbol{k}}^{1, n+1}\right]$ and $\left[d_{\boldsymbol{j}, \boldsymbol{k}}^{2, n+1}\right]$ from $\left[\boldsymbol{d}_{\boldsymbol{j}, \boldsymbol{k}}^{d i v, n+1}\right]$ using the change of basis between $\left\{\left(\psi_{j, k}^{d}\right)^{\prime}\right\}$ and $\left\{\psi_{j, k}^{0 d}\right\}[23]$.

As the matrices $\mathcal{A}_{\delta t}^{0 d}$ and $\mathcal{A}_{\delta t}^{0}$ are inverted once for all before starting the algorithm, Step 1 is thus only a matrix-matrix multiplication. If $j$ denotes the maximal space resolution, i.e, $N=\left(2^{j}+1\right)^{2}$ grid points, the theoretical complexity of this step is at most $O\left(2^{3 j}\right)$. Step 2 corresponds to $\boldsymbol{v}_{j}^{n+1}=\mathbb{P}_{j}^{d i v, 0}\left(\tilde{\boldsymbol{v}}_{j}^{n+1}\right)$, this is solved with a preconditioned conjugate gradient method. Since $\mathbb{M}_{d i v, 0}$ is part of $\mathbb{M}_{d i v}$, which is the stiffness matrix of the 2D Laplacian operator on the scalar wavelet basis of $\left(V_{j}^{d} \otimes V_{j}^{d}\right)$, the complexity of Step 2 can not exceed the complexity of a Poisson solver on the same wavelet basis (quasi-linear complexity [8, 10, 22]). The last step is a change of basis, with a linear complexity. Finally the theoretical complexity of the whole algorithm is at most $O\left(2^{3 j}\right)$, if the whole set of wavelet coefficients is considered. In practice the effective complexity is much lower, due to the sparse structure of both the wavelet coefficients and wavelet operators. 
3.4. Stability and consistency analysis. This section analyzes the stability and consistency of the modified projection method for the unsteady Stokes problem. For sake of simplicity we take $\boldsymbol{f}^{n}=0$ in (3.8) and suppose that $\boldsymbol{v}^{n}, \tilde{\boldsymbol{v}}^{n} \in\left(H_{0}^{1}(\Omega)\right)^{2}$ are regular enough.

THEOREM 3.1. The modified projection method (3.7) and (3.8) for the Stokes equations is unconditionally stable.

Proof. To prove the stability of scheme (3.8), a standard energy estimate will be used with $\tilde{\boldsymbol{v}}^{n+1}$ as test function, thanks to the Dirichlet boundary conditions on $\tilde{\boldsymbol{v}}^{n+1}$. Taking the inner product of equation (3.8) with $2 \tilde{\boldsymbol{v}}^{n+1}$, we obtain

$$
\left\|\tilde{\boldsymbol{v}}^{n+1}\right\|_{L^{2}}^{2}+\left\|\tilde{\boldsymbol{v}}^{n+1}-\boldsymbol{v}^{n}\right\|_{L^{2}}^{2}-\left\|\boldsymbol{v}^{n}\right\|_{L^{2}}^{2}+2 \nu \delta t\left\|\nabla \tilde{\boldsymbol{v}}^{n+1}\right\|_{L^{2}}^{2}=0 .
$$

Since $\tilde{\boldsymbol{v}}^{n+1}=\boldsymbol{v}^{n+1}+\nabla \Phi^{n+1}$, which is an $L^{2}$-orthogonal decomposition in $H_{0}^{1}(\Omega)^{2}$, equation (3.15) simplifies:

$$
\left\|\boldsymbol{v}^{n+1}\right\|_{L^{2}}^{2}+\left\|\boldsymbol{v}^{n+1}-\boldsymbol{v}^{n}\right\|_{L^{2}}^{2}+2\left\|\nabla \Phi^{n+1}\right\|_{L^{2}}^{2}-\left\|\boldsymbol{v}^{n}\right\|_{L^{2}}^{2}+2 \nu \delta t\left\|\nabla \tilde{\boldsymbol{v}}^{n+1}\right\|_{L^{2}}^{2}=0 .
$$

which leads to:

$$
\left\|\boldsymbol{v}^{n+1}\right\|_{L^{2}}^{2}-\left\|\boldsymbol{v}^{n}\right\|_{L^{2}}^{2}+2\left\|\nabla \Phi^{n+1}\right\|_{L^{2}}^{2}+2 \nu \delta t\left\|\nabla \tilde{\boldsymbol{v}}^{n+1}\right\|_{L^{2}}^{2} \leq 0
$$

and completes the proof.

The convergence of the method is a consequence of the following theorem:

THEOREM 3.2. Let $\boldsymbol{v}$ be a smooth solution of Stokes equations with smooth initial data $\boldsymbol{v}_{0}(x)$ and let $\boldsymbol{v}_{\delta t}$ be the numerical solution of the semi-discrete modified projection method (3.7) and (3.8), then:

$$
\begin{aligned}
& \left\|\boldsymbol{v}-\boldsymbol{v}_{\delta t}\right\|_{L^{\infty}\left([0, T] ; L^{2}\right)} \leq C_{1} \delta t \\
& \left\|\nabla \boldsymbol{v}-\nabla \boldsymbol{v}_{\delta t}\right\|_{L^{\infty}\left([0, T] ; L^{2}\right)} \leq C_{2} \delta t^{1 / 2} .
\end{aligned}
$$

Remark that the modified projection method has the same convergence order than the backward Euler scheme, which means that the projection step preserves the time discretization order. A similar result holds for more accurate time discretization schemes like the Crank-Nicolson one.

Proof. Let $\boldsymbol{v}^{n+1}$ be the solution of (3.7) and (3.8) computed from $\boldsymbol{v}^{n}(x)=$ $\boldsymbol{v}(x, n \delta t)$. Let $\boldsymbol{\epsilon}^{n+1}=\boldsymbol{v}(x,(n+1) \delta t)-\boldsymbol{v}^{n+1}$ be the consistency error. Thus, $\boldsymbol{\epsilon}^{n+1}$ is linked to $\tilde{\boldsymbol{v}}^{n+1}$ by:

$$
\tilde{\boldsymbol{v}}^{n+1}=\boldsymbol{v}(\cdot,(n+1) \delta t)+\nabla \Phi^{n+1}-\boldsymbol{\epsilon}^{n+1} .
$$

Replacing (3.17) in (3.6) we obtain:

$$
-\boldsymbol{\epsilon}^{n+1}+\nu \delta t \Delta \boldsymbol{\epsilon}^{n+1}+\delta t \nabla p^{n+1}+\boldsymbol{v}(x,(n+1) \delta t)-\boldsymbol{v}^{n}-\nu \delta t \Delta \boldsymbol{v}(x,(n+1) \delta t)=0 .
$$

Using now the Taylor series expansions:

$$
\begin{aligned}
\boldsymbol{v}(x,(n+1) \delta t) & =\boldsymbol{v}^{n}(x)+\delta t \frac{\partial \boldsymbol{v}}{\partial t}(x, n \delta t)+O\left(\delta t^{2}\right), \\
\Delta \boldsymbol{v}(x,(n+1) \delta t) & =\Delta \boldsymbol{v}(x, n \delta t)+O(\delta t)
\end{aligned}
$$


then:

$\boldsymbol{v}(x,(n+1) \delta t)-\boldsymbol{v}^{n}(x)-\nu \delta t \Delta \boldsymbol{v}(x,(n+1) \delta t)=\delta t\left[\frac{\partial \boldsymbol{v}}{\partial t}(x, n \delta t)-\nu \Delta \boldsymbol{v}(x, n \delta t)\right]+O\left(\delta t^{2}\right)$.

Since $\boldsymbol{v}$ is the exact solution of Stokes equation, we have $\frac{\partial \boldsymbol{v}}{\partial t}-\nu \Delta \boldsymbol{v}=-\nabla p$, and (3.18) rewrites:

$$
-\boldsymbol{\epsilon}^{n+1}+\nu \delta t \Delta \boldsymbol{\epsilon}^{n+1}=\delta t \nabla\left[p(x, n \delta t)-p^{n+1}\right]+O\left(\delta t^{2}\right)
$$

By definition, $\boldsymbol{\epsilon}^{n+1} \in\left(H_{0}^{1}(\Omega)\right)^{2}$ and is divergence-free: $\nabla \cdot \boldsymbol{\epsilon}^{n+1}=0$. Taking $-\boldsymbol{\epsilon}^{n+1}$ as a test function in (3.19) yields:

$$
\left\|\epsilon^{n+1}\right\|_{L^{2}}^{2}+\nu \delta t\left\|\nabla \boldsymbol{\epsilon}^{n+1}\right\|_{L^{2}}^{2}=O\left(\delta t^{2}\right)\left(\int_{\Omega} \boldsymbol{\epsilon}^{n+1}\right) .
$$

Since $\Omega=[0,1]^{2}$ is bounded, then $\left|\int_{\Omega} \epsilon^{n+1}\right| \leq\left\|\epsilon^{n+1}\right\|_{L^{2}}$ and

$$
\left\|\epsilon^{n+1}\right\|_{L^{2}}^{2}+\nu \delta t\left\|\nabla \epsilon^{n+1}\right\|_{L^{2}}^{2} \leq C \delta t^{2}\left\|\epsilon^{n+1}\right\|_{L^{2}}
$$

which implies

$$
\left\|\boldsymbol{\epsilon}^{n+1}\right\|_{L^{2}} \leq C \delta t^{2}, \quad\left\|\nabla \boldsymbol{\epsilon}^{n+1}\right\|_{L^{2}} \leq \frac{C}{\sqrt{\nu}} \delta t^{3 / 2}
$$

The method is then consistent and using the stability result, it converges with a first order convergence in time. The second estimate follows from the fact that one can prove that $\left\|\nabla \boldsymbol{v}^{n}\right\|_{L^{2}}$ is bounded using (3.5).

The spacial consistency error depends on the regularity $s$ of the solution $\boldsymbol{v}$ and the approximation order $r$ provided by spaces $\mathbf{V}_{j}^{d i v, 0}(2.11)$, sucht that $0 \leq s \leq r-1$.

THEOREM 3.3. Let $\boldsymbol{v}$ be a s-smooth solution of Stokes equations with smooth initial data $\boldsymbol{v}_{0}(x)$ and let $\boldsymbol{v}_{j}^{n}$ be the wavelet numerical solution in $\mathbf{V}_{j}^{\text {div, } 0}$ of the semidiscrete modified projection method (3.11) computed from $\boldsymbol{v}_{j}^{0}=\mathbb{P}_{j}^{d i v, 0}\left(\boldsymbol{v}_{0}\right)$, then:

$$
\begin{aligned}
& \left\|\boldsymbol{v}-\boldsymbol{v}_{j}^{n}\right\|_{L^{\infty}\left([0, T] ; L^{2}\right)} \leq C_{1}\left(\delta t+2^{-j s}\right), \\
& \sqrt{\delta t}\left\|\nabla \boldsymbol{v}-\nabla \boldsymbol{v}_{j}^{n}\right\|_{L^{\infty}\left([0, T] ; L^{2}\right)} \leq C_{2}\left(\delta t+2^{-j(s-1)}\right) .
\end{aligned}
$$

where the constant $C_{1}, C_{2}$ are independant of $\delta t, n$ and $j$.

Proof. The proof can be viewed as a modified Cea's lemma. Let $\boldsymbol{\epsilon}_{j}^{n}$ and $\tilde{\boldsymbol{\epsilon}}_{j}^{n}$ be the errors defined by $\boldsymbol{\epsilon}_{j}^{n}=\boldsymbol{v}^{n}-\boldsymbol{v}_{j}^{n}$ and $\tilde{\boldsymbol{\epsilon}}_{j}^{n}=\tilde{\boldsymbol{v}}^{n}-\tilde{\boldsymbol{v}}_{j}^{n}$, where $\tilde{\boldsymbol{v}}^{n}, \boldsymbol{v}^{n}$ are the solutions of (3.8) and $\tilde{\boldsymbol{v}}_{j}^{n}, \boldsymbol{v}_{j}^{n}$ the solutions of (3.11), computed at resolution $j$ and time $n \delta t$. Remark that $\nabla \cdot \epsilon_{j}^{n}=0$.

At step $n$, we suppose that $\boldsymbol{v}_{j}^{n}=\mathbb{P}_{j}^{d i v, 0}\left(\boldsymbol{v}^{n}\right)$, and we study the consistency error $\boldsymbol{\epsilon}_{j}^{n+1}$ provided by (3.11). We have:

$$
\begin{aligned}
& a_{\delta t}\left(\tilde{\boldsymbol{v}}^{n+1}, \boldsymbol{w}\right)=\int_{\Omega} \boldsymbol{v}^{n} \boldsymbol{w}, \quad \boldsymbol{v}^{n+1}=\mathbb{P}^{d i v, 0}\left(\tilde{\boldsymbol{v}}^{n+1}\right), \quad \forall \boldsymbol{w} \in\left(H_{0}^{1}(\Omega)\right)^{2}, \\
& a_{\delta t}\left(\tilde{\boldsymbol{v}}_{j}^{n+1}, \boldsymbol{w}_{j}\right)=\int_{\Omega} \boldsymbol{v}_{j}^{n} \boldsymbol{w}_{j}, \quad \boldsymbol{v}_{j}^{n+1}=\mathbb{P}_{j}^{d i v, 0}\left(\tilde{\boldsymbol{v}}_{j}^{n+1}\right), \quad \forall \boldsymbol{w}_{j} \in \mathbf{V}_{j}^{d} .
\end{aligned}
$$


Taking $\boldsymbol{w}=\boldsymbol{w}_{j} \in \mathbf{V}_{j}^{d}$, we obtain:

$$
a_{\delta t}\left(\tilde{\boldsymbol{\epsilon}}_{j}^{n+1}, \boldsymbol{w}_{j}\right)=\int_{\Omega} \boldsymbol{\epsilon}_{j}^{n} \boldsymbol{w}_{j} \quad \text { and } \quad \boldsymbol{\epsilon}_{j}^{n+1}=\mathbb{P}^{d i v, 0}\left(\tilde{\boldsymbol{v}}^{n+1}\right)-\mathbb{P}_{j}^{d i v, 0}\left(\tilde{\boldsymbol{v}}_{j}^{n+1}\right) .
$$

Now, considering $\boldsymbol{w}_{j} \in\left(\mathbf{V}_{j}^{d i v, 0}\right)^{\perp} \cap \boldsymbol{V}_{j}^{d}$, then $\boldsymbol{w}_{j}=\nabla \phi$ for some $\phi$ and one has $\int_{\Omega} \boldsymbol{\epsilon}_{j}^{n} \boldsymbol{w}_{j}=0$, which implies: $a_{\delta t}\left(\tilde{\boldsymbol{\epsilon}}_{j}^{n+1}, \boldsymbol{w}_{j}\right)=0$.

Moreover since $\boldsymbol{v}_{j}^{n}=\mathbb{P}_{j}^{d i v, 0}\left(\boldsymbol{v}^{n}\right)$, then $\int_{\Omega} \boldsymbol{v}^{n} \boldsymbol{w}_{j}=\int_{\Omega} \boldsymbol{v}_{j}^{n} \boldsymbol{w}_{j}$ for all $\boldsymbol{w}_{j} \in \mathbf{V}_{j}^{d i v, 0}$, which leads to $a_{\delta t}\left(\tilde{\boldsymbol{\epsilon}}_{j}^{n+1}, \boldsymbol{w}_{j}\right)=0$. Finally, we have

$$
a_{\delta t}\left(\tilde{\boldsymbol{\epsilon}}_{j}^{n+1}, \boldsymbol{w}_{j}\right)=0, \quad \forall \boldsymbol{w}_{j} \in \boldsymbol{V}_{j}^{d}
$$

Taking $\boldsymbol{w}_{j}=\boldsymbol{u}_{j}-\tilde{\boldsymbol{v}}_{j}^{n+1}$ in (3.22), which satisfies $\boldsymbol{w}_{j} \in \boldsymbol{V}_{j}^{d}$ for any $\boldsymbol{u}_{j} \in \boldsymbol{V}_{j}^{d}$, we get:

$$
0=a_{\delta t}\left(\tilde{\boldsymbol{\epsilon}}_{j}^{n+1}, \boldsymbol{u}_{j}-\tilde{\boldsymbol{v}}_{j}^{n+1}\right)=a_{\delta t}\left(\tilde{\boldsymbol{\epsilon}}_{j}^{n+1}, \boldsymbol{u}_{j}-\tilde{\boldsymbol{v}}^{n+1}\right)+a_{\delta t}\left(\tilde{\boldsymbol{\epsilon}}_{j}^{n+1}, \tilde{\boldsymbol{v}}^{n+1}-\tilde{\boldsymbol{v}}_{j}^{n+1}\right),
$$

which can be rewritten as:

$$
a_{\delta t}\left(\tilde{\boldsymbol{\epsilon}}_{j}^{n+1}, \tilde{\boldsymbol{\epsilon}}_{j}^{n+1}\right)=a_{\delta t}\left(\tilde{\boldsymbol{\epsilon}}_{j}^{n+1}, \tilde{\boldsymbol{v}}^{n+1}-\boldsymbol{u}_{j}\right) .
$$

From the continuity of the bilinear for $a_{\delta t}(3.10)$, we deduce that:

$$
a_{\delta t}\left(\tilde{\boldsymbol{\epsilon}}_{j}^{n+1}, \tilde{\boldsymbol{v}}^{n+1}-\boldsymbol{u}_{j}\right) \leq(1+\nu \delta t)\left\|\tilde{\boldsymbol{\epsilon}}_{j}^{n+1}\right\|_{H^{1}}\left\|\tilde{\boldsymbol{v}}^{n+1}-\boldsymbol{u}_{j}\right\|_{H^{1}}, \quad \forall \boldsymbol{u}_{j} \in \boldsymbol{V}_{j}^{d} .
$$

and

$a_{\delta t}\left(\tilde{\boldsymbol{\epsilon}}_{j}^{n+1}, \tilde{\boldsymbol{v}}^{n+1}-\boldsymbol{u}_{j}\right) \leq\left\|\tilde{\boldsymbol{\epsilon}}_{j}^{n+1}\right\|_{L^{2}}\left\|\tilde{\boldsymbol{v}}^{n+1}-\boldsymbol{u}_{j}\right\|_{L^{2}}+\nu \delta t\left\|\tilde{\boldsymbol{\epsilon}}_{j}^{n+1}\right\|_{L^{2}}\left\|\tilde{\boldsymbol{v}}^{n+1}-\boldsymbol{u}_{j}\right\|_{H^{2}}, \quad \forall \boldsymbol{u}_{j} \in \boldsymbol{V}_{j}^{d}$,

On the other hand, using the Poincaré-Friedrichs [19] inequality leads to:

$$
\nu \delta t\left\|\tilde{\boldsymbol{\epsilon}}_{j}^{n+1}\right\|_{H^{1}}^{2} \lesssim a_{\delta t}\left(\tilde{\boldsymbol{\epsilon}}_{j}^{n+1}, \tilde{\boldsymbol{\epsilon}}_{j}^{n+1}\right)
$$

and we also have:

$$
\left\|\tilde{\boldsymbol{\epsilon}}_{j}^{n+1}\right\|_{L^{2}}^{2} \leq a_{\delta t}\left(\tilde{\boldsymbol{\epsilon}}_{j}^{n+1}, \tilde{\boldsymbol{\epsilon}}_{j}^{n+1}\right)
$$

Then, (3.23) gives:

$$
\sqrt{\nu \delta t}\left\|\tilde{\boldsymbol{\epsilon}}_{j}^{n+1}\right\|_{H^{1}} \lesssim\left\|\tilde{\boldsymbol{v}}^{n+1}-\boldsymbol{u}_{j}\right\|_{H^{1}}, \quad \forall \boldsymbol{u}_{j} \in \boldsymbol{V}_{j}^{d} .
$$

For $\boldsymbol{u}_{j}=\mathbf{P}_{j}^{d}\left(\tilde{\boldsymbol{v}}^{n+1}\right)$ (projection onto $\left.\boldsymbol{V}_{j}^{d}\right)$, the usual Jackson's inequality yields:

$$
\sqrt{\nu \delta t}\left\|\tilde{\boldsymbol{\epsilon}}_{j}^{n+1}\right\|_{H^{1}} \lesssim 2^{-(j-1) s}\left\|\tilde{\boldsymbol{v}}^{n+1}\right\|_{H^{s}} \lesssim 2^{-(j-1) s}\left\|\tilde{\boldsymbol{v}}^{0}\right\|_{H^{s}}
$$

where we have used the stability properties $(3.15,3.16)$.

Similarly, one has:

$$
\left\|\tilde{\boldsymbol{\epsilon}}_{j}^{n+1}\right\|_{L^{2}} \lesssim\left(2^{-j s}+\nu \delta t 2^{-j(s-2)}\right)\left\|\tilde{\boldsymbol{v}}^{0}\right\|_{H^{s}} .
$$

Since $\mathbf{V}_{j}^{d i v, 0}=\mathbf{V}_{j}^{d} \cap \mathcal{H}_{d i v, 0}(\Omega)$, see [23], we have $\mathbb{P}_{j}^{d i v, 0}\left(\tilde{\boldsymbol{v}}_{j}^{n+1}\right)=\mathbb{P}^{d i v, 0}\left(\tilde{\boldsymbol{v}}_{j}^{n+1}\right)$ and the two errors are linked by the relation:

$$
\boldsymbol{\epsilon}_{j}^{n+1}=\mathbb{P}^{d i v, 0}\left(\tilde{\boldsymbol{\epsilon}}_{j}^{n+1}\right) \Rightarrow\left\|\boldsymbol{\epsilon}_{j}^{n+1}\right\|_{L^{2}} \leq\left\|\tilde{\boldsymbol{\epsilon}}_{j}^{n+1}\right\|_{L^{2}} \text { and }\left\|\boldsymbol{\epsilon}_{j}^{n+1}\right\|_{H^{1}} \leq\left\|\tilde{\boldsymbol{\epsilon}}_{j}^{n+1}\right\|_{H^{1}}
$$


Finally, to prove (3.20) it suffices to write, for all $n$ :

$$
\begin{aligned}
\left\|\boldsymbol{v}-\boldsymbol{v}_{j}^{n}\right\|_{L^{2}}=\left\|\boldsymbol{v}-\boldsymbol{v}^{n}+\boldsymbol{v}^{n}-\boldsymbol{v}_{j}^{n}\right\|_{L^{2}} & \leq\left\|\boldsymbol{v}-\boldsymbol{v}^{n}\right\|_{L^{2}}+\left\|\boldsymbol{v}^{n}-\boldsymbol{v}_{j}^{n}\right\|_{L^{2}} \\
& \lesssim\left(\delta t+2^{-j s}+\nu \delta t 2^{-j(s-2)}\right) \\
& \lesssim\left(\delta t+2^{-j s}\right),
\end{aligned}
$$

using theorem 3.2 and $(3.25)$, as $\nu 2^{-j(s-2)} \leq 1$. Similar arguments allow to prove (3.21) using the $H^{1}$ norm, which completes the proof. $\mathrm{\square}$

3.5. Example. To investigate the convergence rates of the wavelet modified projection method for the unsteady Stokes problem (3.1), two numerical tests are conducted: the first one to evaluate the time discretization error and the second one to evaluate the spatial discretization error. As exact solution, we used:

$$
\left\{\begin{array}{l}
v_{1}(x, y, t)=\frac{1}{8 \pi^{2} \nu}\left(1-e^{-8 \pi^{2} \nu t}\right)[\cos (2 \pi x) \sin (2 \pi y)-\sin (2 \pi y)] \\
v_{2}(x, y, t)=\frac{-1}{8 \pi^{2} \nu}\left(1-e^{-8 \pi^{2} \nu t}\right)[\sin (2 \pi x) \cos (2 \pi y)-\sin (2 \pi x)] \\
p(x, y, t)=0.5 e^{-t}[\cos (2 \pi x)-\cos (2 \pi y)]
\end{array}\right.
$$

This solution satisfies Dirichlet homogeneous boundary conditions $\boldsymbol{v}_{\mid \partial \Omega}=0$, where $\Omega=[0,1]^{2}$. The right-hand side term $\boldsymbol{f}$ is computed appropriately to ensure that (3.26) is the exact solution of system (3.1). The $1 \mathrm{D}$ wavelet generators of $\left(V_{j}^{1}, \tilde{V}_{j}^{1}\right)$ are biorthogonal splines with $r=\tilde{r}=3$, which means that the approximation order of $V_{j}^{0}$ is 2 ; this corresponds to a bidimensional approximation order of at most 2 .

For the time discretization we chose two implicit methods that are easy to implement: backward-Euler and Crank-Nicholson schemes. We recall that the order of these schemes are 1 and 2 respectively. However, any accurate numerical scheme would have been be used without restriction on the order. Tab. 3.1 shows the $L^{2}$-error between the exact solution projected onto $\mathbf{V}_{j}$ (with a space resolution fixed at $j=10$ and $\nu=2^{-j}$ ), and the numerical solution of (3.11), in terms of the discretization time step $\delta t$. As expected (theorem 3.2), the time discretization convergence rate is close

\begin{tabular}{||l||l|l|l|l||l||}
\hline \multicolumn{7}{|c|}{ Backward-Euler } \\
\hline$\delta t$ & 0.1 & 0.05 & 0.025 & 0.0125 & Order \\
\hline$L_{2}$-error & $25.24 E^{-3}$ & $12.70 E^{-3}$ & $63.71 E^{-4}$ & $31.90 E^{-4}$ & 0.9922 \\
\hline \hline \multicolumn{5}{|c|}{ Crank-Nicholson } \\
\hline$\delta t$ & 0.1 & 0.05 & 0.025 & 0.0125 & Order \\
\hline$L_{2}$-error & $30.09 E^{-6}$ & $71.72 E^{-7}$ & $14.48 E^{-7}$ & $16.91 E^{-8}$ & 2.0031 \\
\hline
\end{tabular}

TABLE 3.1

Time discretization $\ell^{2}$-error according to the time step $\delta$, for final time $T=2$.

to the theoretical order (1 or 2) of the chosen time scheme.

For the evaluation of the spatial discretization error, we considered a regular grid. Following theorem 3.3, we have to chose a time step very small compared to the spatial resolution: we took $\delta t=5.10^{-4}$ and $\nu=2^{-10}$. Tab. 3.2 shows the spatial error at grid points for the simulation final time $T=2$. Remark that, as the solution is $\mathcal{C}^{\infty}$, the convergence rate given by Tab. 3.2 saturates to the number of vanishing moments 


\begin{tabular}{||l||l|l|l|l||l||}
\hline \multicolumn{5}{|c||}{ Backward-Euler } \\
\hline$j$ & 6 & 7 & 8 & 9 & Order \\
\hline$L_{\infty}$-error & $32.11 \mathrm{E}^{-7}$ & $80.23 \mathrm{E}^{-8}$ & $20.03 \mathrm{E}^{-8}$ & $49.79 \mathrm{E}^{-9}$ & 2.026 \\
\hline$L_{2}$-error & $19.84 \mathrm{E}^{-7}$ & $49.18 \mathrm{E}^{-8}$ & $12.26 \mathrm{E}^{-8}$ & $30.49 \mathrm{E}^{-9}$ & 2.023 \\
\hline \hline$H^{1}$-error & $13.38 \mathrm{E}^{-5}$ & $57.99 \mathrm{E}^{-6}$ & $26.49 \mathrm{E}^{-6}$ & $15.51 \mathrm{E}^{-6}$ & 1.045 \\
\hline \hline \multicolumn{6}{|c||}{ Crank-Nicholson } \\
\hline$j$ & 6 & 7 & 8 & 9 & Order \\
\hline$L_{\infty}$-error & $32.11 \mathrm{E}^{-7}$ & $80.27 \mathrm{E}^{-8}$ & $20.06 \mathrm{E}^{-8}$ & $50.17 \mathrm{E}^{-9}$ & 2.000 \\
\hline$L_{2}$-error & $19.84 \mathrm{E}^{-7}$ & $49.21 \mathrm{E}^{-8}$ & $12.29 \mathrm{E}^{-8}$ & $30.72 \mathrm{E}^{-9}$ & 2.020 \\
\hline$H^{1}$-error & $13.38 \mathrm{E}^{-5}$ & $58.00 \mathrm{E}^{-6}$ & $26.54 \mathrm{E}^{-6}$ & $14.05 \mathrm{E}^{-6}$ & 1.088 \\
\hline
\end{tabular}

TABLE 3.2

Spatial discretization errors at grid points according to the resolution $j$, for final time $T=2$.

of our wavelet family (equal to 2 in our spline approximation for the $L^{2}$-error), and we lose one order for the $H^{1}$-error.

\section{Modified projection method for unsteady Navier-Stokes equations.}

4.1. Divergence-free wavelet schemes for Navier-Stokes equations. Divergence-free wavelet schemes for the numerical resolution of Navier-Stokes equations were first introduced by Deriaz and Perrier [13, 14], in the case of periodic boundary conditions. In this section, we will consider Dirichlet boundary conditions, and we will present a new scheme for Navier-Stokes equations, in the projection method setting $[7,35]$, but replacing the original projection step by the divergence-free wavelet Leray projector, as in Section 3.1.

4.2. Temporal discretization of Navier-Stokes equations. Since the original works of Chorin [7] and Temam [35], the projection method has become highly popular for the numerical resolution of Navier-Stokes equations in velocity pressure formulation, notably with physical, such as no-slip, boundary conditions. Several versions of the projection method exist, according to the chosen pressure boundary condition [1]. Without loss of generality, we focus here on the second order boundary approximation in time, called projection method with accurate pressure boundary condition [15]. The principle (time) steps of this method is summarized bellow [15]:

- Prediction step: knowing $\boldsymbol{v}^{n} \in \mathcal{H}_{\text {div }, 0}(\Omega)$, compute an intermediate velocity field $\boldsymbol{v}^{*}$ such that:

$$
\left\{\begin{array}{l}
\frac{\boldsymbol{v}^{*}-\boldsymbol{v}^{n}}{\delta t}+\left(\boldsymbol{v}^{n+1 / 2} \cdot \nabla\right) \boldsymbol{v}^{n+1 / 2}=\nu \Delta \frac{\boldsymbol{v}^{*}+\boldsymbol{v}^{n}}{2} \\
\boldsymbol{v}^{*}=0, \text { on } \partial \Omega
\end{array}\right.
$$

with

$$
\left(\boldsymbol{v}^{n+1 / 2} \cdot \nabla\right) \boldsymbol{v}^{n+1 / 2}=\frac{3}{2}\left(\boldsymbol{v}^{n} \cdot \nabla\right) \boldsymbol{v}^{n}-\frac{1}{2}\left(\boldsymbol{v}^{n-1} \cdot \nabla\right) \boldsymbol{v}^{n-1}
$$

- Correction step: project $\boldsymbol{v}^{*}$ onto the divergence-free functions space to get 


$$
\begin{aligned}
& \boldsymbol{v}^{n+1} \in \mathcal{H}_{\text {div }, 0}(\Omega): \\
& \left\{\begin{array}{l}
\boldsymbol{v}^{n+1}=\boldsymbol{v}^{*}-\delta t \nabla p^{n+1 / 2}, \\
\nabla \cdot \boldsymbol{v}^{n+1}=0, \\
\nabla p^{n+1 / 2} \cdot \boldsymbol{n}=-\boldsymbol{n} \cdot\left[\nabla \times\left(\nabla \times \boldsymbol{v}^{*}\right)\right], \text { on } \partial \Omega,
\end{array}\right.
\end{aligned}
$$

with $\nabla p^{n+1 / 2}=\frac{3}{2} \nabla p^{n}-\frac{1}{2} \nabla p^{n-1}$.

In classical approaches $[1,7,24,35]$ the computation of the velocity $\boldsymbol{v}^{n+1}$ needs first to solve a Poisson equation:

$$
\delta t \Delta p^{n+1 / 2}=\nabla \cdot \boldsymbol{v}^{*},
$$

with suitable boundary condition. Since $\boldsymbol{v}^{*}$ and $\boldsymbol{v}^{n+1}$ vanish on the boundary $\partial \Omega$, we should have $\nabla p^{n+1 / 2}=0$ on $\partial \Omega$, which means:

$$
\nabla p^{n+1 / 2} \cdot \boldsymbol{n}=0 \quad \text { and } \quad \nabla p^{n+1 / 2} \cdot \boldsymbol{\tau}=0 \text { on } \partial \Omega .
$$

Any Poisson solver can not incorporate both boundary conditions (4.5) at the same time, the problem becoming overdetermined. In practice, to solve (4.4), only the Neumann boundary condition for the pressure $\nabla p^{n+1 / 2} \cdot \boldsymbol{n}=0$ is considered $[15,24]$, which leads to a loss of accuracy at the boundary [15]. Moreover, preferring one of these two boundary conditions (4.5) distorts the Dirichlet homogeneous boundary condition satisfied by $\boldsymbol{v}$ :

$$
\nabla p^{n+1 / 2} \cdot \boldsymbol{n}=0 \Rightarrow \boldsymbol{v}^{n+1} \cdot \boldsymbol{\tau}=\nabla p^{n+1 / 2} \cdot \boldsymbol{\tau} \neq 0
$$

and

$$
\nabla p^{n+1 / 2} \cdot \boldsymbol{\tau}=0 \Rightarrow \boldsymbol{v}^{n+1} \cdot \boldsymbol{n}=\nabla p^{n+1 / 2} \cdot \boldsymbol{n} \neq 0
$$

As done for the Stokes equations, the objective of the following paragraph will be to define a divergence-free wavelet based modified projection method for NavierStokes equation that moreover satisfies (4.5), this will be a major difference with the methods based on a Poisson solver.

Analyzing differently the problem, one can take advantage of the boundary conditions like (4.5), the Helmholtz-Hodge decomposition in $\left(H_{0}^{1}(\Omega)\right)^{2}$ and the new construction of divergence-free wavelets with boundary conditions, to derive a new correction step for (4.1). Indeed, let $\Phi^{n+1 / 2}$ be a scalar potential in $L^{2}(\Omega)$ satisfying:

$$
\tilde{\boldsymbol{v}}^{n+1}=\boldsymbol{v}^{n+1}+\nabla \Phi^{n+1 / 2}, \text { with } \tilde{\boldsymbol{v}}^{n+1} \in\left(H_{0}^{1}(\Omega)\right)^{2} .
$$

Substituting this change of variable in a Crank-Nicolson time scheme, (4.1) and (4.3) are replaced by the following new prediction and correction steps for the intermediate velocity $\tilde{\boldsymbol{v}}^{n+1}$ and pressure computation:

- Prediction step:

$$
\left\{\begin{array}{l}
\frac{\tilde{\boldsymbol{v}}^{n+1}-\boldsymbol{v}^{n}}{\delta t}+\left(\boldsymbol{v}^{n+1 / 2} \cdot \nabla\right) \boldsymbol{v}^{n+1 / 2}=\nu \Delta \frac{\tilde{\boldsymbol{v}}^{n+1}+\boldsymbol{v}^{n}}{2}, \\
\tilde{\boldsymbol{v}}^{n+1}=0, \text { on } \partial \Omega .
\end{array}\right.
$$


- Correction step:

$$
\left\{\begin{array}{l}
\boldsymbol{v}^{n+1}=\mathbb{P}^{d i v, 0}\left(\tilde{\boldsymbol{v}}^{n+1}\right) \\
p^{n+1 / 2}=\frac{1}{\delta t} \Phi^{n+1 / 2}-\frac{\nu}{2} \Delta \Phi^{n+1 / 2}
\end{array}\right.
$$

where $\mathbb{P}^{d i v, 0}$ denotes the $L^{2}$-orthogonal projector from $\left(H_{0}^{1}(\Omega)\right)^{2}$ onto $\mathcal{H}_{\text {div }, 0}(\Omega)$ introduced in Section 2.2. Remark that the Navier-Stokes formulation (4.7) is no more than a change of variables, whereas the classical projection method is an operator splitting, which implies a loss of precision.

The spatial discretization uses a Wavelet Galerkin formulation as for the Stokes equations in Section 3.1: the components of $\boldsymbol{v}^{n}$ and $\boldsymbol{v}^{*}$ are searched in the form of a finite wavelet series defined by (3.9). Following [1, 24], the nonlinear term $\left(\boldsymbol{v}^{n+1 / 2}\right.$. $\nabla) \boldsymbol{v}^{n+1 / 2}(4.2)$ is computed explicitly with finite differences on the mesh grid points, and this explicit treatment imposes a CFL condition on the time step, see [13]. The order of this finite difference scheme has to be at least equal to the approximation order of the wavelet approximation (namely the parameter $r$ ), to not reduce the global spatial accuracy of the method.

4.3. Exemple. To investigate the accuracy and spatial convergence rate of the modified projection method (4.7) and (4.8), we performed a convergence study on regular grids. The exact solution is that given in (3.26) with $\nu=1$ (using a suitable forcing term). As for the Stokes problem, the $1 \mathrm{D}$ wavelet generators of $\left(V_{j}^{1}, \tilde{V}_{j}^{1}\right)$ are biorthogonal splines with $r=\tilde{r}=3$, which corresponds to a bidimensional spatial approximation order of at most 2. We used a time scheme of order 2 (Crank Nicolson), with a time step $\delta t=5.10^{-4}$, chosen small enough such that the error induced by the time discretization is negligible with respect to the spatial discretization error. In Fig 4.1 we plotted the $L^{2}, L^{\infty}$ and $H^{1}$ norms of the velocity error in terms of the grid step in $\log -\log$ scale, at the simulation final time $T=1$. As it was stated in Th.3.3 for the Stokes problem, since the solution is $\mathcal{C}^{\infty}$, Fig 4.1 gives a spatial convergence rate which saturates to the number of vanishing moments of our wavelet family (equal to 2 in our spline approximation for the $L^{2}$-error), and we lose one order for the $H^{1}$-error.

4.4. Lid driven cavity flow. To validate the divergence-free wavelet projection method on Navier-Stokes equations, we focus on the classical lid-driven cavity problem on regular grids. This problem has been investigated by many authors since the pioneer work of [3, 18]. Recently, Bruneau and Saad [4] provide new simulations at high Reynolds numbers, obtaining highly accurate benchmark results, using a multigrid solver with a special emphasis on the discretization of the convection term, for which a high space resolution is used: $j=10$ or $j=11$.

The objective in this section is to compare the results obtained with the scheme (4.7)-(4.8) to those of $[3,4,18]$, in order to evaluate the accuracy and performance of this new method.

The governing equations are the Navier-Stokes equations (1.1) on $\Omega=] 0,1\left[^{2}\right.$ with 


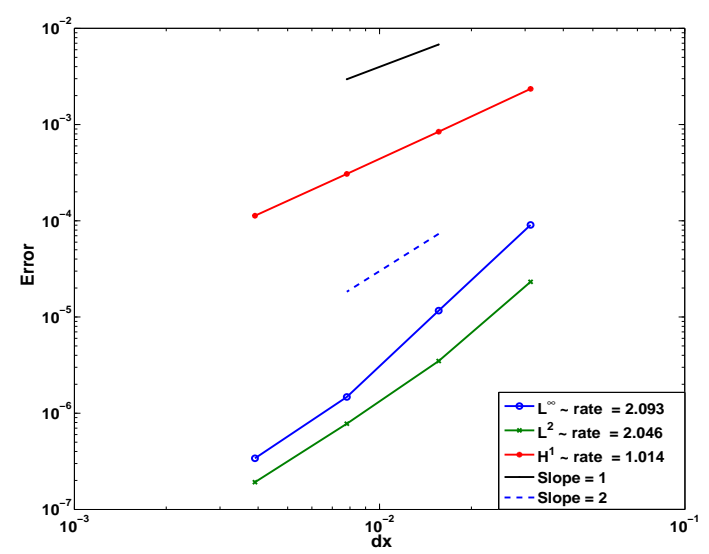

FIGURE 4.1. Spatial errors at grid points according to $\delta x$ (log-log scale), scheme (4.7) and (4.8) for the simulation final time $T=1$.

the (free-slip) non homogenous boundary condition on $\Gamma=\partial \Omega$ :

$$
\boldsymbol{v}_{\mid \Gamma}=\boldsymbol{g}(x, y, t)=\left\{\begin{array}{lll}
\boldsymbol{g}(x, 1, t)=(-1,0) & \forall t \in] 0, T[, \quad \forall x \in] 0,1[ \\
\boldsymbol{g}(x, y, t)=(0,0) & \forall t \in] 0, T[, & \forall(x, y) \in \Gamma, y \neq 1 .
\end{array}\right.
$$

The initial velocity being chosen as $\boldsymbol{v}(x, y, 0)=0$ in $\Omega$ and the Reynolds number corresponds to $R e=\frac{1}{\nu}$ in equation (1.1).

Boundary condition (4.9) means that for all $t>0$, the edge scaling functions and wavelet coefficients of $\boldsymbol{v}$ can be computed once from those of $\boldsymbol{g}$. In practice, to incorporate (4.9) on $\boldsymbol{v}^{n}$, it suffices to impose $\tilde{\boldsymbol{v}}_{\mid \Gamma}=\boldsymbol{g}$ in (4.7) and replace $\mathbb{P}^{d i v, 0}$ by $\mathbb{P}^{d i v}$ in (4.8). Since the new horizontal velocity $\tilde{v}_{1}$ does not satisfy homogeneous Dirichlet boundary condition, an homogenization technique is used for this component to solve (4.7), see [21]. The wavelet basis generators of $\left(V_{j}^{1}, \tilde{V}_{j}^{1}\right)$ are biorthogonal splines with three vanishing moments for both space family: $r=\tilde{r}=3$. The advection term $\left(\boldsymbol{v}^{n+1 / 2} \cdot \nabla\right) \boldsymbol{v}^{n+1 / 2}$ is computed with a finite difference method of order 3 on a regular grid, the same approximation order as that of the wavelet spaces. For the steady convergence state, Fig. 4.2 shows the plot of the middle horizontal and vertical profiles of the velocity obtained with the present method for $j=7$ spatial resolution and $R e=1000$, and compared to the results of [4] obtained with $j=10$ spatial resolution. The vorticity contour and the divergence-free scaling functions coefficient for this Reynolds number $R e=1000$ are plotted on Fig. 4.3 and their values again confirm the convergence of the method. Tab. 4.1 and Tab. 4.2 show the values of these profiles computed with $j=7$ and $j=8$ spatial resolution for present method, $j=10$ spatial resolution for Bruneau and Saad [4], compared to pioneers results of [18] and [3]. In [3], the spatial discretization is done using a spectral method with $N=160$ Chebyshev polynomials.

For the simulation at moderate Reynolds number $R e=10000$, to avoid corner problem, a regularized velocity $\boldsymbol{v}(x, 1, t)=\left(-16 x^{2}(1-x)^{2}, 0\right)$ is used. The value $R e=$ 10000 is a critical test case, many studies were performed to see for instance if a steady solution is achieved or not, or if it has periodic behavior [4]. The results obtained with 
the divergence-free wavelet based projection method prove that it remains stable and give rise to a numerical solution. Fig 4.5 shows the divergence-free scaling function coefficients contours, for the simulation time $T=80$, which correspond to the contours of the stream function coefficients on the scalar multiresolution analysis $V_{j}^{1} \otimes V_{j}^{1}$ $(j=8)$. The associated divergence-free wavelet coefficients isovalues are also plotted on Fig. 4.5, which, in this case, gives evidence of the sparsity of the solution, in the wavelet representation. This sparse repartition of the wavelet coefficients is confirmed by Fig. 4.6 which produces the repartition of the divergence-free wavelet coefficients greater, in absolute value, than a thresholding parameter $\epsilon$.
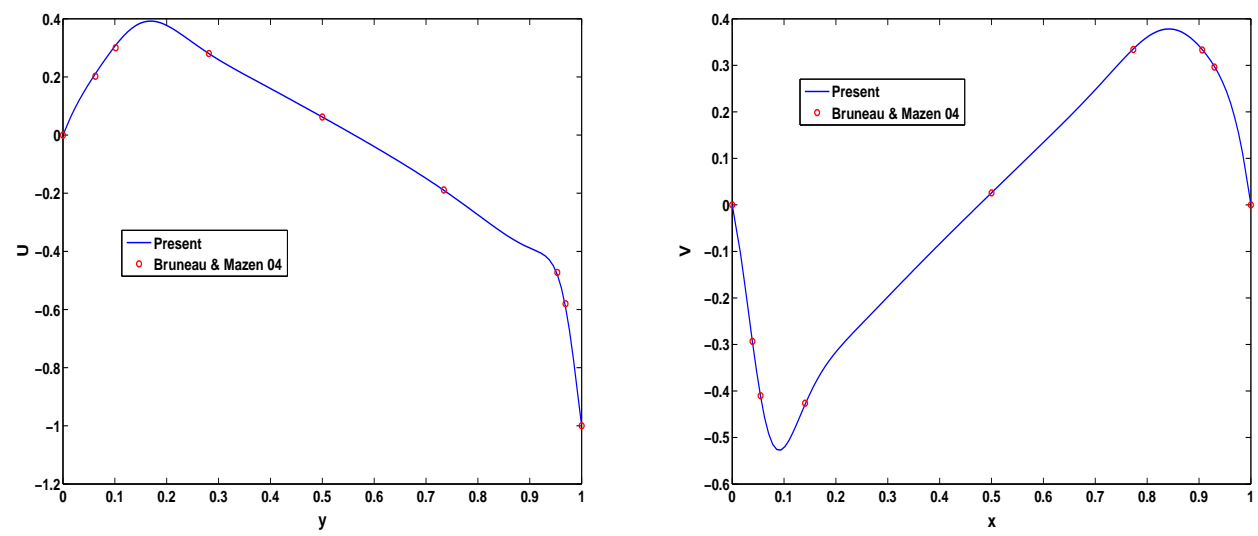

FIGURE 4.2. Steady state horizontal velocity $v_{1}$ (left) and vertical velocity $v_{2}$ (right) profiles in the middle of the cavity. Solid line (present work) and circle (Bruneau and Saad [4]): Re = 1000 and $j=7$.

\begin{tabular}{|l|l|l|l|l|l|l|}
\hline$y$ & $v_{1}, j=7$ & $v_{1}, j=8$ & $v_{1}, j=9$ & $v_{1}$, Ref. [3] & $v_{1}$, Ref. [4] & $v_{1}$, Ref. [18] \\
\hline 1 & -1.0000 & -1.0000 & -1.0000 & -1.0000 & -1.0000 & -1.0000 \\
\hline 0.9688 & -0.5850 & -0.5809 & -0.5815 & -0.5808 & -0.5803 & -0.5749 \\
\hline 0.9531 & -0.4756 & -0.4726 & -0.4722 & -0.4723 & -0.4723 & -0.4660 \\
\hline 0.7344 & -0.1901 & -0.1887 & -0.1878 & -0.1886 & -0.1886 & -0.1871 \\
\hline 0.5000 & 0.0618 & 0.0617 & 0.0618 & 0.0620 & 0.0620 & 0.0608 \\
\hline 0.2813 & 0.2797 & 0.2800 & 0.2795 & 0.2803 & 0.2804 & 0.2780 \\
\hline 0.1016 & 0.3091 & 0.3020 & 0.3010 & 0.3004 & 0.3002 & 0.2973 \\
\hline 0.0625 & 0.2116 & 0.2013 & 0.2021 & 0.2023 & 0.2022 & 0.2019 \\
\hline 0.0000 & 0.0000 & 0.0000 & 0.0000 & 0.0000 & 0.0000 & 0.0000 \\
\hline
\end{tabular}

TABLE 4.1

Velocity $v_{1}$ values in the middle of the cavity at the steady state for $R e=1000$.

Since the wavelet based numerical schemes provide sparse representations of the Navier-Stokes solutions [32], an important issue to improve the numerical complexity should be to implement dynamical adaptive techniques. At present, the Navier-Stokes simulation for the lid driven cavity needs about $2 s$ of CPU time to run one iteration, using $512^{2}$ grid points on the square $[0,1]^{2}$, with a Matlab code on a conventional computer. 


\begin{tabular}{|l|l|l|l|l|l|l|}
\hline$x$ & $v_{2}, j=7$ & $v_{2}, j=8$ & $v_{2}, j=9$ & $v_{2}, \operatorname{Ref}[3]$ & $v_{2}, \operatorname{Ref}[4]$ & $v_{2}, \operatorname{Ref}[18]$ \\
\hline 0.0000 & 0.0000 & 0.0000 & 0.0000 & 0.0000 & 0.0000 & 0.0000 \\
\hline 0.0391 & -0.3086 & -0.2968 & -0.2937 & -0.2936 & -0.2933 & -0.2766 \\
\hline 0.0547 & -0.4267 & -0.4137 & -0.4073 & -0.4103 & -0.4101 & -0.3918 \\
\hline 0.1406 & -0.4253 & -0.4257 & -0.4266 & -0.4264 & -0.4263 & -0.4266 \\
\hline 0.5000 & 0.0266 & 0.0259 & 0.0254 & 0.0257 & 0.0258 & 0.0252 \\
\hline 0.7734 & 0.3359 & 0.3340 & 0.3331 & 0.2803 & 0.2804 & 0.2780 \\
\hline 0.9062 & 0.3364 & 0.3333 & 0.3324 & 0.3339 & 0.3339 & 0.3307 \\
\hline 0.9297 & 0.2998 & 0.2967 & 0.2959 & 0.2962 & 0.2962 & 0.2901 \\
\hline 1.0000 & 0.0000 & 0.0000 & 0.0000 & 0.0000 & 0.0000 & 0.0000 \\
\hline
\end{tabular}

TABLE 4.2

Velocity $v_{2}$ values in the middle of the cavity at the steady state for $R e=1000$.
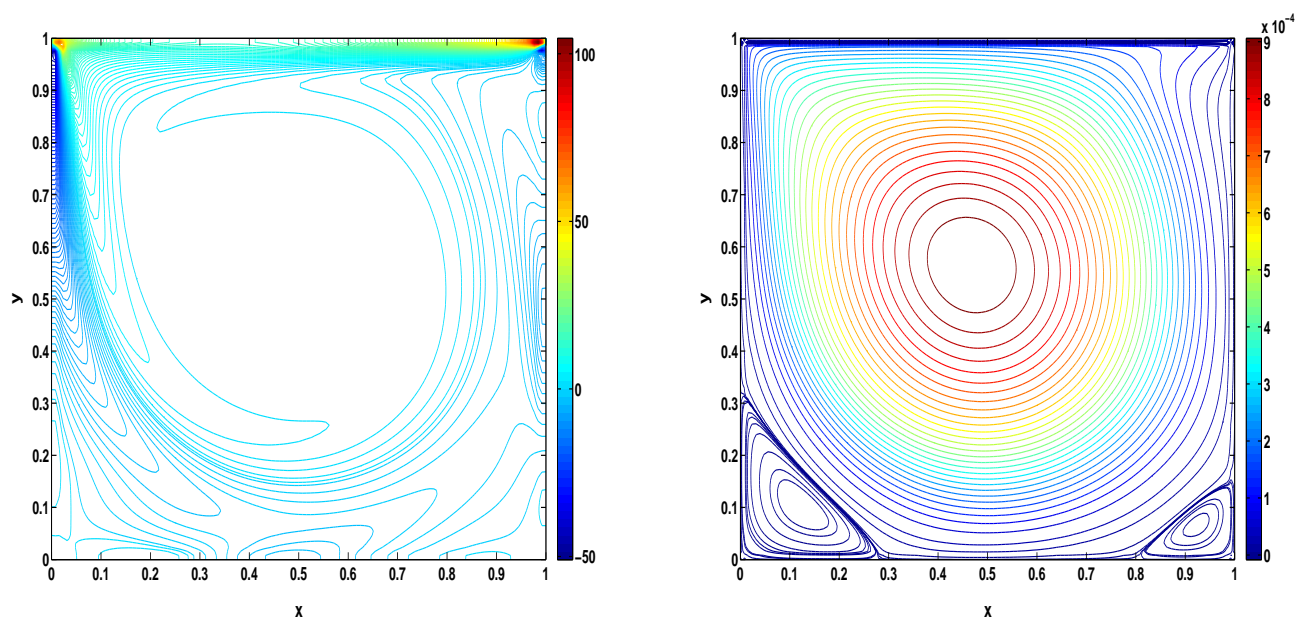

FIGURE 4.3. Vorticity contour (left) and divergence-free scaling function coefficients contour (right). Steady state for $R e=1000$ and $j=7$.

Adaptive procedure should be considered with the present schemes (4.7) and (4.8), which is confirmed by Fig. 4.4 where only almost $1 \%$ of the divergence-free wavelet coefficients are retained for small values of $\epsilon$. To implement adaptive method on schemes (4.7) and (4.8), first notice that (4.7) is a classical elliptic equation for the velocity $\tilde{\boldsymbol{v}}^{n+1}$, for which optimal adaptive wavelet methods have been designed [8], linear with respect to the number of significant wavelet coefficients, i.e. great that some small $\epsilon$. The key-point at that time is the computation of the (explicit) nonlinear term, which for the moment is computed on the regular grid points. For (4.8), the Leray-Hopf projection also reduces to Laplace-Dirichlet problem, in wavelet basis: as previously, this part can also benefit from adaptive schemes developed by [8]. The only points to be considered are first the operator between the divergence-free wavelet coefficients $\left[d_{\boldsymbol{j}, \boldsymbol{k}}^{d i v, n}\right]$ and the "standard" wavelet one $\left[d_{\boldsymbol{j}, \boldsymbol{k}}^{1, n}\right]$ and $\left[d_{\boldsymbol{j}, \boldsymbol{k}}^{2, n}\right]$, which is diagonal (same indices), see [23]. The second point concerns the boundary: to avoid a loss of accuracy, even in adaptive scheme, the same number of edge wavelet functions has to 


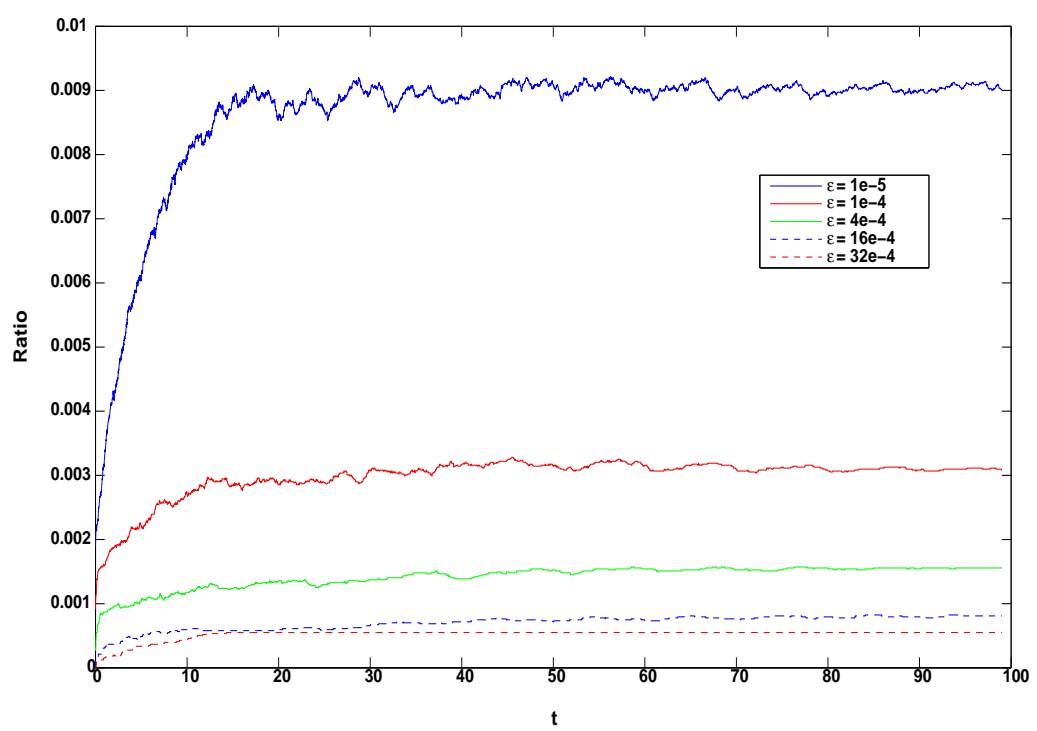

FIGURE 4.4. Evolution in time of the ratio of divergence-free wavelet coefficients up to a fixed $\epsilon$. The maximal space resolution is $j=8$, the coarse decomposition level is $j_{\text {min }}=3$ and $R e=1000$.
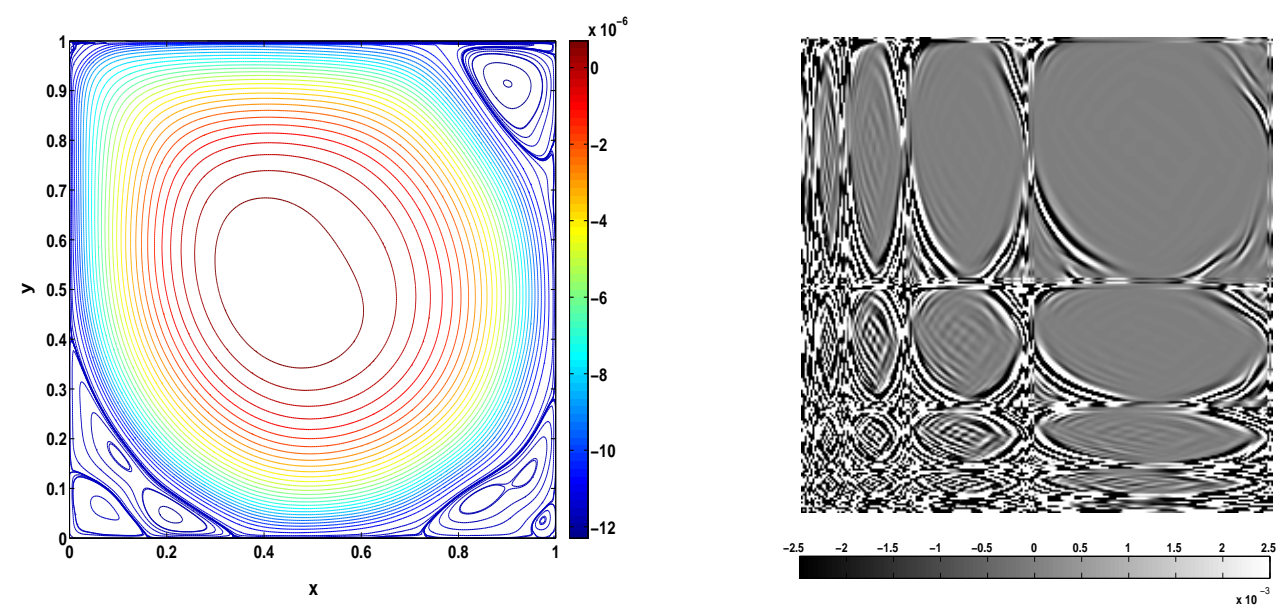

FIGURE 4.5. Contour of divergence-free scaling function coefficients at $j=8$ (left) and divergence-free wavelet coefficients isovalues (right) for a coarse decomposition level $j_{\text {min }}=3$. The simulation final time is $T=80$ and $R e=10000$.

be kept at each level (or scale index). This point is well documented in reference [10].

\section{Conclusion.}

In this paper we have constructed a divergence-free wavelet based projection method for the numerical resolution of Stokes and Navier-Stokes equations with Dirichlet boundary conditions. Stability and consistency of the method were proved for the unsteady Stokes equations and verified numerically for the Navier-Stokes equa- 


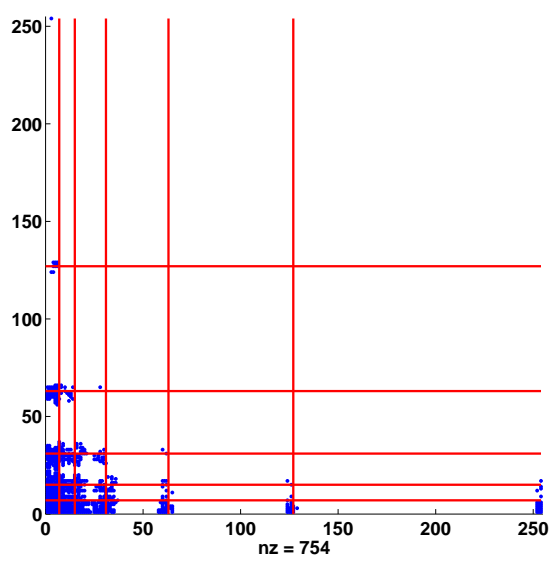

(a) $\epsilon=10^{-4}$.

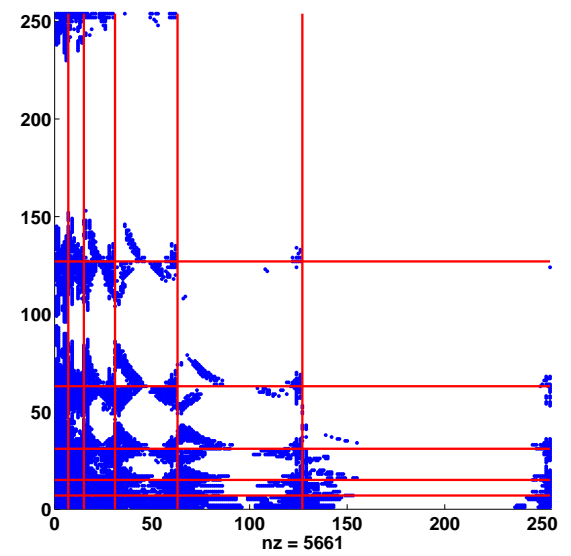

(c) $\epsilon=10^{-6}$.

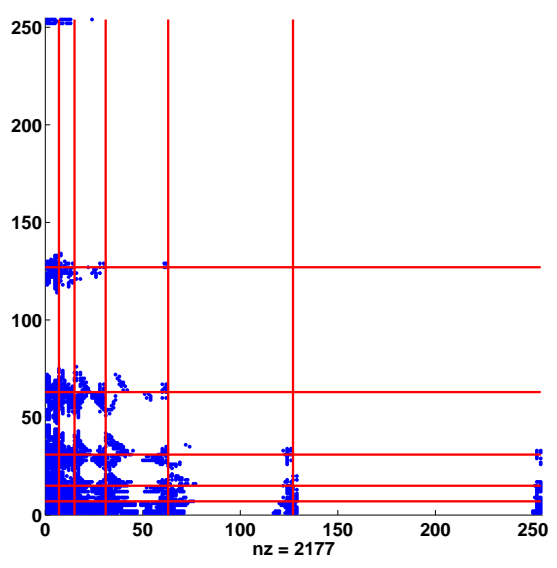

(b) $\epsilon=10^{-5}$.

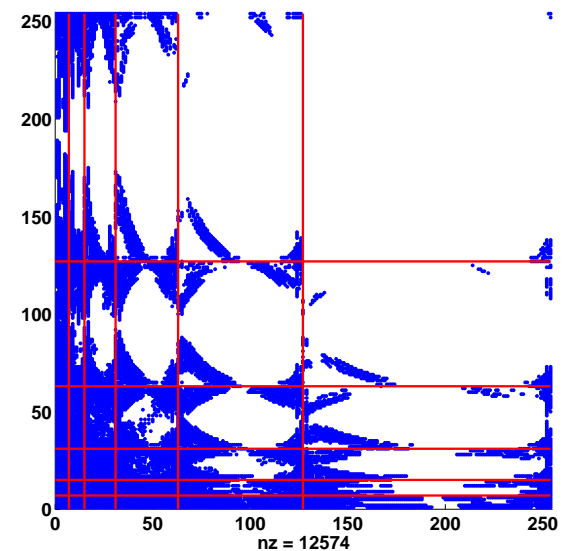

(d) $\epsilon=10^{-7}$.

FIGURE 4.6. Divergence-free wavelet coefficients with absolute values greater than epsilon for a maximal space resolution $j=8$ and a coarse decomposition level $j_{\text {min }}=3$. The simulation final time is $T=80$ and $R e=10000$.

tions, with application to the benchmark problem of the lid-driven cavity flow, using regular grids.

The main interest of the method lies in the correction step where the Dirichlet boundary condition on the divergence-free solution is exactly satisfied, without imposing boundary conditions on the pressure field, which is not the case in classical approaches. An important issue to even more reduce the computational complexity, is to develop an adaptive method, taking advantage of the compression property provided by wavelet bases, which, for elliptic problems, leads to optimal numerical solvers [8]. An extension to dimension three of the present method is on-going: a 3D divergence-free and curl-free wavelet based Helmholtz-Hodge decomposition on the hypercube already exists [22]. Its incorporation in the correction step of a modified 
3D projection method will be the subject of a forthcoming paper.

\section{REFERENCES}

[1] J. B. Bell, P. Colella, H. M. Glaz, A second-order projection method for the incompressible Navier-Stokes equations, J. Comput. Phys., 85 (1989) pp. 257-283.

[2] G. BeYLKIN, On the representation of operator in bases of compactly supported wavelets, SIAM J. Numer. Anal., 6 (1992), pp. 1716-1740.

[3] O. Botella, R. Peyret, Benchmark spectral results on the lid-driven cavity flow, Comput. Fluids, 27 (1998), pp. 421-433.

[4] C.-H. Bruneau, M. SAad, The 2D lid-driven cavity problem revised, Comput. Fluids, 35 (2006), pp. 326-348.

[5] C. Canuto, R. Masson, Stabilized wavelet approximations of the Stokes problem, Math. of Comp., 70 (2001), pp. 1397-1416.

[6] P. Charton, V. Perrier, A Pseudo-Wavelet Scheme for the Two-Dimensional Navier-Stokes Equations, Comp. Appl. Math., 15 (1996), pp. 137-157.

[7] A.J. Chorin, Numerical simulation of the Navier-Stokes equation, Math. Comp., 22 (1968), pp. $745-762$.

[8] A. Cohen, Numerical Analysis of Wavelet Methods, Elsevier, 2003.

[9] A. Cohen, I. Daubechies, P. Vial, Wavelets on the Interval and Fast Wavelet Transforms, Appl. Comput. Harmon. Anal., 1 (1993), pp. 54-81.

[10] A. Cohen, R. Masson, Wavelet methods for second order elliptic problems - preconditioning and adaptivity, SIAM J. Sci. Comp., 21(1999), pp. 1006-1026.

[11] W. Dahmen, K. Urban, J. Vorloeper, Adaptive Wavelet Methods-Basic Concepts and Applications to the Stokes Problem, Wavelet Analysis-Twenty Years Developments, Ding-Xuan Zhou ed., World Scientific, New Jersey, (2002), pp. 39-80.

[12] E. Deriaz, V. Perrier, Orthogonal Helmholtz decomposition in arbitrary dimension using divergence-free and curl-free wavelets, Appl. Comput. Harmon. Anal., 26 (2009), pp. 249269.

[13] E. Deriaz, V. PERrier, Direct Numerical Simulation of Turbulence using divergence-free wavelets, SIAM Multis. Model. and Simul., 7 (2008), pp. 1101-1129.

[14] E. Deriaz, V. Perrier, Divergence-free and curl-free wavelets in 2D and 3D, application to turbulent flows, J. of Turbulence, 7 (2006), pp. 1-37.

[15] W. E, J. Guo-Liu , Projection Method I: Convergence and Numerical Boundary Layers, SIAM J. Numer. Anal., 32 (1995), pp. 1017-1057.

[16] M. FARGE , Wavelet transforms and their applications to turbulence, Ann. Review of Fluid Mechanics., 24(1992), pp. 395-457.

[17] J. Frohlich, K. Schneider, Numerical Simulation of Decaying Turbulence in an Adaptive Wavelet Basis, Appl. Comput. Harm. Anal, 3 (1996), pp. 393-397.

[18] U.Ghia, K. N. GhiA, C. T. Shin, High-Re solutions for incompressible flows using NavierStokes equations and a multigrid method, J. Comput. Phys., 48 (1982), pp. 387-411.

[19] V. Girault, P.A. Raviart, Finite element methods for Navier-Stokes equations, SpringerVerlag Berlin, 1986.

[20] A. Jouini, P.G. Lemarié-Rieusset, Analyse multirésolution biorthogonale sur l'intervalle et applications, Annales de l'I.H.P. Section C, 10 (1993), pp. 453-476.

[21] S. Kadri-Harouna, Ondelettes pour la prise en compte de conditions aux limites en turbulence incompressible, Phd thesis, Grenoble University, 2010.

[22] S. Kadri-Harouna, V. Perrier, Helmholtz-Hodge Decomposition on $[0,1]^{d}$ by Divergence-free and Curl-free Wavelets, Curves and Surfaces, proc of the 7th International Conference, Avignon, France, June 24-30, 2010, Boissonnat, J.-D.; Chenin, P.; Cohen, A.; Gout, C.; Lyche, T.; Mazure, M.-L.; Schumaker, L. (Eds.), Lecture Notes in Computer Science series, 6920, Springer (2012), pp. 311-329.

[23] S. Kadri-Harouna, V. Perrier, Effective construction of divergence-free wavelets on the square, J. of Computational and Applied Math, 240, (2013), pp. 74-86.

[24] J. KIm, P. MoIn, Application of a fractional-step method to incompressible Navier-Stokes equations, J. Comp. Phys., 59 (1985), pp. 308-323.

[25] P. G. LemariÉ-RIEusset, Analyses multi-résolutions non orthogonales, commutation entre projecteurs et dérivation et ondelettes vecteurs à divergence nulle, Revista Matemática Iberoamericana, 8 (1992), pp. 221-236.

[26] J.-G. LiU, J. LiU, R. Pego, Stable and accurate pressure approximation for unsteady incom- 
pressible viscous flow, J. Comput. Phys., 229 (2010), pp. 3428-3453.

[27] R. MAsson, Biorthogonal spline wavelets on the interval for the resolution of boundary problems, M3AS 6 (6) 749-791, 1996.

[28] A. S. Orszag, G. S. Patterson JR., Numerical simulation of turbulence, Lecture Notes in Physics, 12(1972), pp. 127-147.

[29] P. Monasse, V. Perrier, Orthogonal Wavelet Bases Adapted For Partial Differential Equations With Boundary Conditions, SIAM J. Math. Anal., 29 (1998), pp. 1040-1065.

[30] S. Muller, Adaptive Multiscale Schemes for Conservation Laws, Lect. Notes Comput. Sci. Eng. vol. 27, Heidelberg: Springer-Verlag, 2003.

[31] A. D. Polyanin, Handbook of Linear Partial Differential Equations for Engineers and Scientists, Chapman \& Hall/CRC, 2002.

[32] K. Schneider, O. Vasilyev, Wavelet methods in computational fluid dynamics, Annu. Rev. Fluid Mech., 42 (2010), pp. 473-503.

[33] R. Stevenson, Divergence-free wavelet bases on the hypercube: Free-slip boundary conditions, and applications for solving the instationary Stokes equations, Math. Comp. 80 (2011), pp. $1499-1523$.

[34] R. Stevenson, Divergence-free wavelets on the hypercube: General boundary conditions, ESI preprint 2419, Erwin SchrŽdinger Institute, Vienna (2013).

[35] R. Temam, Sur l'approximation de la solution des équations de Navier-Stokes par la méthode des pas fractionnaires II , Arch. Rational Mech. Anal. 33 (1969), pp. 377-385.

[36] R. Temam, Navier Stokes Equations, North Holland, New York, 1977.

[37] K. URBAN, Using divergence-free wavelets for the numerical solution of the Stokes problem, AMLI'96: Proceedings of the Conference on Algebraic Multilevel Iteration Methods with Applications University of Nijmegen, The Netherlands, 2 (1996), pp. 261-277.

[38] K. Urban, Wavelet Bases in H(div) and H(curl), Math. Comput., 70 (2000), pp. 739-766.

[39] K. Urban, Wavelets in Numerical Simulation, Springer Berlin, 2002.

[40] C. Wang, J-G. Liu, Convergence of Gauge method for incompressible flow, Math. Comput., 69 (2000), pp. 1385-1407. 